\title{
A computational stochastic multiscale methodology for MEMS structures involving adhesive contact
}

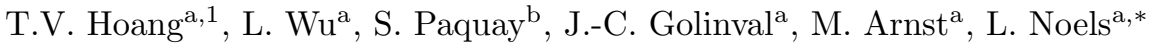 \\ ${ }^{a}$ University of Liège - Department of Aerospace and Mechanical Engineering, \\ Allée de la Découverte 9, Quartier Polytech 1, B-4000 Liège, Belgium. \\ ${ }^{b}$ Open Engineering $S A$, \\ Rue Bois Saint-Jean 15/1, B-4102, Liège (Angler), Belgium.
}

\begin{abstract}
This work aims at developing a computational stochastic multiscale methodology to quantify the uncertainties of the adhesive contact problems due to capillary effects and van der Waals forces in MEMS. Because the magnitudes of the adhesive forces strongly depend on the surface interaction distances, which in turn evolve with the roughness of the contacting surfaces, the involved structural behaviors suffer from a scatter. To numerically predict the probabilistic behaviors of structures involving adhesion, the proposed method introduces stochastic meso-scale random apparent contact forces which can be integrated into a stochastic finite element model. Because the evaluation of their realizations is expensive, a generator for the random apparent contact force using the polynomial chaos expansion is constructed in an efficient way.
\end{abstract}

Keywords: adhesive contact, random surface, multiscale contact, uncertainty quantification

\section{Introduction}

One of the common failures in microelectromechanical systems (MEMS) is stiction $[1,2,3,4,5,6,7,8]$, in which two micro surfaces permanently adhere together, e.g. the stiction failure of micro cantilever beams illustrated in Fig. 1(a). The failure is due to the dominance of the adhesive surface forces, such as van der Waals (vdW) forces and capillary forces, in comparison with the body forces. On the one hand, in humid conditions condensing menisci develop between the contacting hydrophilic surfaces, result into relative negative pressures, and lead to the so-called humid stiction, see Fig. 1(b). On the other hand, in dry environments vdW forces become dominant.

MEMS stiction failure is an uncertain phenomenon as it is experimentally observed in $[4,5,9]$. In the present work, a stochastic multiscale model is developed to quantify the uncertainty of MEMS stiction. The model is developed with three assumptions: (i) the considered source of the scatter in stiction is the randomness of contacting surfaces; (ii) the contacting surfaces are nominally flat with nanometers roughness; (iii) to model the capillary forces, the constant pressure assumption is applied [10].

In the adhesive problems of MEMS, due to the comparability between the surface roughness and the ranges of the adhesive forces, the interaction does not involve the whole surface topography but only its highest asperities, see Fig. 1(b) [2]. Moreover, due to the separation of scales between the ranges of the adhesive forces and the structural displacements, the effective contact regions are much smaller than the structural dimensions. For instance, in cases of micro cantilever beams, the effective contact regions are located only around the crack tips defined as the separating point between the unattached part and attached part of the failed beam, see Fig. 1(a). We can thus define three characteristic length scales:

\footnotetext{
*Corresponding author, Phone: +32436648 26, Fax: +3243669505

Email address: L. Noels@ulg.ac.be (L. Noels)

${ }^{1} \mathrm{PhD}$ candidate at the Belgian National Fund for Education at the Research in Industry and Farming.
} 
(i) The lower length scale $l^{\mathrm{m}}$, typically $\mathcal{O}(1 \mu \mathrm{m})$ for the studied rough surfaces as it will be discussed, measured by the correlation length of the contacting rough surface, which is defined here in this paper as the spatial distance at which the evaluated autocorrelation of the rough surfaces is negligible in comparison with the surface roughness -from the point of view of materials, $l^{\mathrm{m}}$ relates to the grain size of the considered structure;

(ii) The meso-scale length $l^{\text {meso }}$, typically $\mathcal{O}(3 \mu \mathrm{m})$ for the studied beam stiction problem, which is defined as the length on which the variation of the structural displacement, e.g. the displacement of beam central line in case of micro beams, is negligible in comparison with the roughness of the contacting surface (nanometers for MEMS); and

(iii) The upper scale length $l^{\mathrm{M}}$, typically $\mathcal{O}(100 \mu \mathrm{m})$, which is characterized by the size of the considered structures.

Because of the comparability between the length scales $l^{\mathrm{m}}$ and $l^{\mathrm{mes}}$, and of the randomness of contact surfaces, there exist uncertainties in the adhesive behaviors of the micro structures $[1,11,12]$. From the point of view of multi-asperities contact, the randomness of surfaces affects not only the distribution of the asperities heights, but also their spatial arrangement, e.g. around the crack tips see Fig. 1. Indeed, the contact between the rough surfaces does not involve the whole apparent surfaces but only their highest topologies [4]: the physical contact area is only $\mathcal{O}(1 \%)$ of the apparent area. As a result, the physical interaction occurs between a limited number of asperities (typically $\mathcal{O}(100)$ for the meso-scale size contacting surfaces in the studied problem), and therefore the contact behavior is uncertain as discussed in [13]. For micro cantilever beams, the crack lengths, defined as the length of the open, unattached part of the failure configuration, see Fig. 1(a), can suffer from a scatter. The crack length characterizes the adhesion strength, i.e. the shorter the crack length, the higher the exhibited adhesion strength.

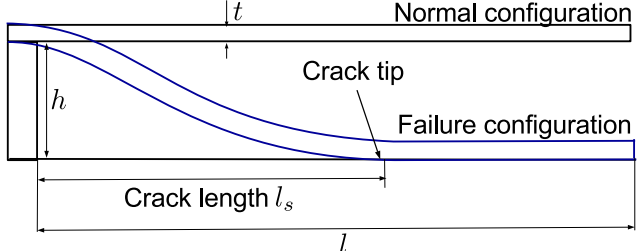

(a) Initial and failure configurations (not on scale).

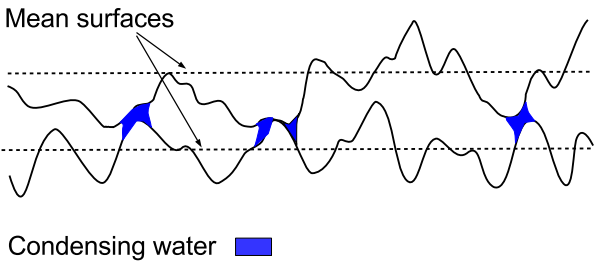

(b) Stiction between two surfaces (not on scale).

Figure 1: The stiction failure of a cantilever beam structure under humid condition: (a) initial configuration and S-shape failure configuration with crack length $l_{s}$, (b) zoom into the contact zone with the condensing water area on the surface topology.

For the adhesive rough surface contact problems of micro structures, because there exist uncertainties which significantly affect micro structures reliability, the scatter on the contact behaviors was already studied in the literature, e.g. in $[1,11,12,14]$. Statistical models to predict the adhesion strength, such as the adhesive surface energy, were developed in these researches. However, in order to quantify not only the adhesive surface energies but also the reliability of the devices involving adhesion, further steps are required. The key step is to be able to predict the probabilistic behavior of structures subjected to adhesive contact forces by accounting for the randomness of contact surfaces. This paper aims at developing a numerical methodology to accomplish this task by coupling a multiscale methodology for micro structures involving adhesive rough surface contact to an uncertainty quantification process. 
In terms of deterministic stiction models, the non-concurrent multiscale method, developed in [7], is considered. The method is developed thanks to the length scales separation between the length scale of the surfaces roughness, $l^{\mathrm{m}}$, and the length scale of the structural displacements, $l^{\mathrm{M}}$. The main ingredient of this multiscale method is to construct the apparent adhesive contact forces, which homogenize the effects of the surface roughness and can be integrated, under the formulas of a contact law, into a finite element (FE) model of the studied structure. Mathematically speaking, the apparent contact force is an average force integrated on the apparent interacting area defining by meso-scale length $l^{\text {meso }}$.

In the literature, two main classes of methods can be found to evaluate the apparent contact forces for the adhesive problems: (i) analytical methods such as the Greenwood and Williamson (GW) approach [15, 16]; and (ii) numerical methods such as FE models [17, 18, 19, 20], molecular dynamics (MD) models [21, 22, 23], or FE-MD hybrid models [24]. The first candidate, the GW approach, uses the statistical parameters of the contact surfaces [25] to deduce the equivalent asperity contact model on which the asperity contact theories $[26,27,28,29]$ are applied to evaluate the contact forces. Due to its simple implementation, this model is widely used. However, the approach requires some cautions $[14,16]$. For instance, it is limited to the cases of deterministic contacts for which the contact surface size effect is not accounted. The second candidate, a full numerical model using either a FE, a MD, or a FE-MD hybrid model, requires a high computational cost, e.g. a FE model needs to discretize the micro-scale size surfaces with a nano-scale mesh size, which limits their applicability when performing uncertainty quantification.

In order to predict the uncertainties of the structural behaviors accounting for the existing randomness in the contact surfaces, the apparent contact forces are considered as random variables. To evaluate realizations of random apparent contact forces, the probabilistic contact model previously developed by the authors in [14], which is characterized by an acceptable computational cost, is considered. The advantage of this method is to account for the surfaces sizes effect on the statistical behavior, which is required for the uncertainty quantification process, when evaluating the apparent contact forces. In order to account for the randomness of contact surfaces, that method estimates the power spectrum density (PSD) functions of the interacting surfaces from their topology measurements, e.g. obtained by the means of the atomic force microscopy (AFM). Applying the random field theory, the realizations of contact surfaces with the size of interest can be generated. For each surface realization, the corresponding apparent contact force is then evaluated by a semi-analytical solution. However, in [14], the evaluated random apparent contact forces were not used in a multiscale FE model because of the difficulty to formulate a stochastic multiscale FE approach, which is the main purpose of the present paper.

In this paper, these apparent adhesive contact forces are integrated as contact laws in a FE code leading to a multiscale model [7]. Taking advantage of the stochastic method of the apparent contact force, and in order to investigate the structural behaviors in a probabilistic way, the multiscale method is coupled with an uncertainty quantification process. The convenient solution is to consider direct Monte Carlo simulations (MCS) [30]. This direct MCS method follows three main steps: (i) constructing a generator for the considered rough contact surfaces using their estimated PSD functions; (ii) for each generated contact surface of the contact zone size, evaluating the corresponding meso-scale apparent contact forces; and (iii) integrating the meso-scale apparent contact forces into a FE model to evaluate the non-deterministic structural behaviors. The direct MCS method evaluates the set of structural behaviors corresponding to the set of the generated surfaces from which the probability of the structural behaviors is identified. This requires a large number of apparent contact forces to be evaluated, and in turn, demands large computational resources. To reduce the computational cost, we consider here a stochastic model in the multiscale method. Although, such method was never developed for adhesive contact problems, we take advantage of previous works [31, 32, 33].

In this stochastic model-based multi-scale method, the apparent adhesive contact forces are considered as a random variable which are efficiently generated by a stochastic model. The latter is built from a limited number of explicitly evaluated apparent contact forces, while as many as required contact forces can be generated at a reduced cost. In order to construct the stochastic model, the apparent force vs. distance curves are parametrized via an adhesive analytical contact function in order to be represented by vectors of parameters. The stochastic model is then constructed to generate the random parameters vectors. To construct the stochastic model approximating the probability of the target random vector, common methods choose a labeled distribution, e.g. uniform, Gaussian or log-normal distribution ..., whose parameters are 
estimated using the input set of explicitly evaluated samples. An alternative method, based on the generalized polynomial chaos expansion (gPCE), see e.g. [34, 35, 36, 37, 38], constructs a transformation from a labeled random vector, whose distribution is known, to the target random vector. The second method is more flexible than the first one in approximating the studied random vectors as it can represent any second order random vectors and is therefore adopted in this work. The construction of the gPCE-based stochastic model is based on Rosenblatt isoprobabilistic transformation [39] and is highly efficient in terms of computational cost.

This paper is structured as follows. In Section 2, a probabilistic multiscale framework for adhesive contact problems is developed following 4 stages: (i) the introduction of the stochastic multiscale framework using random apparent contact forces, (ii) the lower-scale model: the probabilistic model of random contact surfaces, (iii) the meso-scale model: the evaluation of apparent contact forces accounting for capillary and vdW effects; and (iv) the upper-scale model: the stochastic FE model of the structural behaviors. In Section 3, the methodologies to quantify the randomness of the structural behaviors involving stiction are developed: (i) the direct MCS multiscale method, and (ii) the stochastic model-based multiscale method. The stochastic model of the random apparent contact forces, required in the latter, is then constructed. In Section 4 , the developed methodology is applied to predict the uncertainties of the stiction failure of poly-silicon micro cantilever beams. In this section, a comparison of the two uncertainty propagation methods, the direct MCS method and the stochastic model-based multiscale method, in terms of numerical results and computational efficiency, is also given. In Section 5, the methodology is validated through the comparison of the numerical results with the experimental results reported in $[5,9]$.

\section{Computational probabilistic multiscale framework for adhesive contact problems}

In this section we develop a multiscale framework to evaluate the probabilistic behavior of micro structures involving frictionless adhesive elastic contacts, see e.g. [7, 41, 42]. In order to simplify the numerical evaluation of the contact forces, the contact between two rough surfaces $\left\{\mathcal{S}_{1}, \mathcal{S}_{2}\right\}$ is modeled as the interaction between a plane and an equivalent rough surface, $\mathcal{S}$, whose statistical properties are deduced from those of the contacting surfaces $\left\{\mathcal{S}_{1}, \mathcal{S}_{2}\right\}[40]$.

A random rough contact surface $\mathcal{S}$ is modeled by adding a zero mean random field $z$, discussed in Section 2.2.1, to the mean surface $\overline{\mathcal{S}}$, which corresponds to the contact boundary of the upper scale geometry. For instance, in the case of micro beams, the mean surface $\overline{\mathcal{S}}$ is located at a distance $t / 2$ below the beam neutral surface, with $t$ the beam average thickness, see Fig. 2(a). When considering the homogenization technique, the mean surface $\overline{\mathcal{S}}$ is associated with the contact laws, which are the apparent adhesive contact forces evaluated using the meso-scale contact problem, see Fig. 2(b), and illustrated in Fig. 2(c). The structural scale problem in which the meso-scale apparent adhesive contact forces are integrated to represent the lower scale contact is then efficiently solved by a FE model. The integration of these apparent adhesive contact forces into the FE model is numerically implemented such that each integral point, used to evaluate the equivalent nodal forces and located on $\overline{\mathcal{S}}$, is associated with an apparent adhesive contact force. As these contact forces are random, as illustrated in Fig. 2(c), different integral points are associated with different contact forces.

This section is developed as follows: (i) a stochastic multiscale scheme for adhesive contact problems is derived using the random apparent adhesive contact forces as the scales bridge; (ii) the probabilistic contact model developed in [14] to evaluate these forces due to capillary effects is summarized and enhanced to account for vdW forces; and (iii) a stochastic FE model is applied to solve the upper scale problem, i.e. to study the structural behaviors.

\subsection{Stochastic multiscale scheme for adhesive contact problems}

In this section, a stochastic multi-sale scheme is developed using the random apparent contact forces as the scales bridge and the energy consistency condition to derive the expression of the homogenized contact law. 


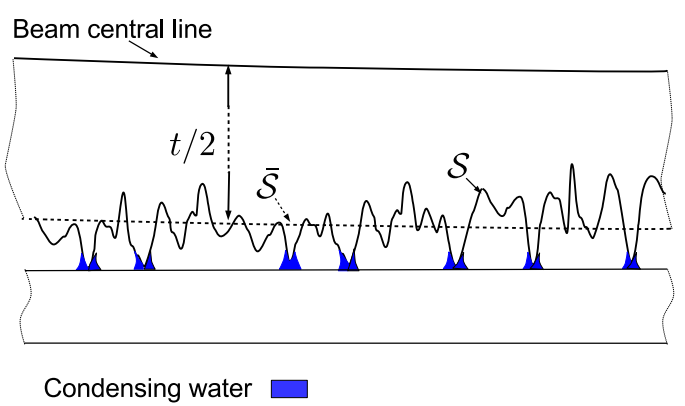

(a) Contact problem (not on scale).

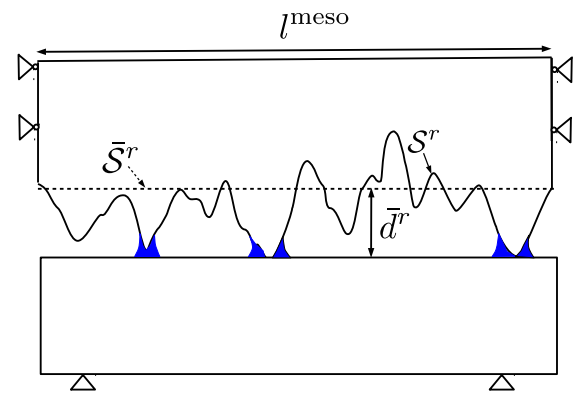

(b) Meso-scale contact problem (not on scale).

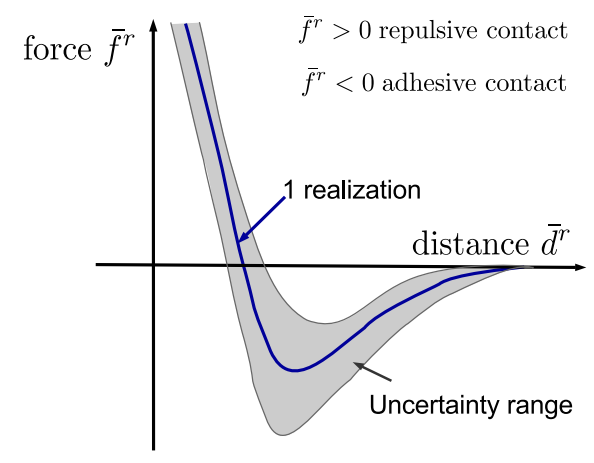

(c) Apparent contact forces.

Figure 2: The multiscale model of adhesive contact problem applied for micro beams. (a) The contact configuration between a rough surface beam and a plane in humid conditions. In this figure, $t$ is the beam thickness. (b) The 1D meso-scale contact problem. (c) The random meso-scale apparent adhesive contact forces.

Upper scale governing equation. At the upper scale, a numerical FE model of the contact behavior replaces the real contact rough surface $\mathcal{S}$ by its mean surface $\overline{\mathcal{S}}$, see Fig. 2(a), and associates this mean surface with the upper scale contact forces $f$ representing the lower scale rough surface adhesive contact. Let us define $u$ and $u_{\overline{\mathcal{S}}}$ as respectively the upper scale structural displacements of the whole structure and the upper scale normal displacements of the mean surface $\overline{\mathcal{S}}$. At the upper scale of the structural behavior, the weak formulation governing equation of the elastic structure subjected to the upper scale contact forces $f$ applied on the mean surface $\overline{\mathcal{S}}$ is given by

$$
\delta U=\delta W^{\mathrm{c}}\left(\mathcal{S}(\theta), u_{\overline{\mathcal{S}}}\right)+\delta W^{\mathrm{a}},
$$

where $\mathcal{S}(\theta)$ refers to a realization of the random surface $\mathcal{S}$ with $\theta$ defined on the probability space $(\Omega, \mathfrak{F}, P)$, and $\delta U, \delta W^{\mathrm{a}}, \delta W^{\mathrm{c}}$ are respectively the internal virtual work, the external virtual work of applied forces, and the external virtual work of contact forces resulting from the adhesive contact. The external virtual work $\delta W^{\mathrm{c}}$ is evaluated from the upper scale contact forces as

$$
\delta W^{\mathrm{c}}=\int_{\overline{\mathcal{S}}} f\left(\mathcal{S}(\theta), u_{\overline{\mathcal{S}}}\right) \delta u_{\overline{\mathcal{S}}} \mathrm{d} \overline{\mathcal{S}} .
$$

Meso-scale apparent adhesive contact force. Let $\mathbf{x}^{r} \in \overline{\mathcal{S}}$, with $r=1, \ldots, N_{p}$, be the $N_{p}$ integral points located on the mean surface $\overline{\mathcal{S}}$ of the upper scale numerical model used to evaluate the external virtual work of contact forces, Eq. (2). Each integral point is associated with a sub-contact domain which involves a part, $\mathcal{S}^{r}$, of the rough surface $\mathcal{S}$ with size $l_{1}^{\text {meso }} \times l_{2}^{\text {meso }}$ and centered at $\mathbf{x}^{r}$, see Fig. 2(b). For isotropic cases with $2 \mathrm{D}$ contact structural scale model, $l_{1}^{\text {meso }}=l_{2}^{\text {meso }}=l^{\text {meso }}$. As discussed previously, the meso-scale 
length $l^{\text {meso }}$ is determined to satisfy the condition that the variation of the displacement $u_{\overline{\mathcal{S}}^{r}}$ of the mean surface $\overline{\mathcal{S}}^{r}$ is negligible in comparison with the roughness of the surfaces, see analyses in Section 4.2. With that assumption, let us define $\bar{d}^{r}$ the normal distance from the mean surface $\overline{\mathcal{S}}^{r}$ to the encountering plane. The meso-scale apparent contact force, defined as the average of the lower-scale normal contact force, $f^{\mathrm{m}}$, applied on the rough surface $\mathcal{S}^{r}$ and for which the displacement of the mean surface $\overline{\mathcal{S}}^{r}$ is prescribed to be parallel with the plane at a distance $\bar{d}^{r}$, see Fig. 2(b), is given by

$$
\bar{f}^{r}\left(\bar{d}^{r}, \theta\right)=\frac{1}{A_{\overline{\mathcal{S}}^{r}}} \int_{\mathcal{S}^{r}(\theta)} f^{\mathrm{m}}\left(\bar{d}^{r}, \theta\right) \mathrm{d} \mathcal{S} .
$$

In this equation $A_{\overline{\mathcal{S}}^{r}}=l_{1}^{\text {meso }} \times l_{2}^{\text {meso }}$ is the area of surface $\overline{\mathcal{S}}^{r}$. A realization of the apparent adhesive contact forces and their uncertainty range are illustrated in Fig. 2(c). For the meso-scale contact problem, the energy consistency requirement is stated as

$$
f\left(\mathbf{x}^{r}\right) \delta u_{\overline{\mathcal{S}}}\left(\mathbf{x}^{r}\right)=\frac{1}{A_{\overline{\mathcal{S}}^{r}}} \int_{\mathcal{S}^{r}(\theta)} f^{\mathrm{m}}\left(\bar{d}^{r}, \theta\right) \delta \bar{d}^{r} \mathrm{~d} \mathcal{S} .
$$

As on the meso-scale contact problem, the displacement of the mean surface is uniform and constrained to be $\delta \bar{d}^{r}=\delta u_{\overline{\mathcal{S}}}$, this last relation (4) is satisfied with the structural scale forces defined as

$$
f\left(\mathbf{x}^{r}\right)=\bar{f}^{r}, \text { with } r=1, \ldots, N_{p}
$$

In the following, the probabilistic contact model developed in [14] for the evaluation of the random apparent contact forces, $\bar{f}^{r}\left(\bar{d}^{r}, \theta\right)$, is first summarized, and a stochastic FE model of the structural behaviors using these forces as the contact laws is then developed.

\subsection{Evaluation of the meso-scale apparent adhesive contact forces}

In order to evaluate the realizations of the random apparent contact force, defined by Eq. (3) and illustrated in Fig. 2(c), three properties have to be accounted for: (i) the topology of the surfaces; (ii) the adhesive interaction; and (iii) the constitutive material laws of the contacting bodies. For the first issue, the probabilistic contact model developed by the authors in [14] estimates the PSD functions of the two involved surfaces $\left\{\mathcal{S}_{1}, \mathcal{S}_{2}\right\}$ from their topology measurements, e.g. using AFM for MEMS surfaces, and then evaluates the equivalent PSD function corresponding to the equivalent surface $\mathcal{S}$. Using the equivalent PSD function, a set of independent identically distributed (IID) equivalent surfaces is generated. To account for the last two issues, a contact model is applied to evaluate the apparent contact forces for the generated surfaces. In the cases of the adhesive contact problems on (relative) hard materials, such as the humid stiction or vdW interaction on poly-silicon surfaces, the semi-analytical method developed in [14] can be applied to evaluate the contact forces with limited computational resources.

\subsubsection{Probabilistic model and random generator of the roughness contacting surfaces}

The normal height distribution of a rough surface $\mathcal{S}(\theta)$, measured from its mean surface, can be modeled as a random field $(Z(\mathbf{x}, \theta), \mathbf{x} \in \mathfrak{D})$ valued in $\mathfrak{R}$, indexed by $\mathfrak{D} \subset \mathfrak{R}^{2}$, and defined on the probability triplet $(\Omega, \mathfrak{F}, P)$. In $[14]$ we have modeled the rough surface as a zero-mean stationary isotropic Gaussian random field for which the autocorrelation function $r_{Z}$ is given by [43]

$$
\begin{aligned}
r_{Z}\left(\tau_{1}, \tau_{2}\right) & =\mathbb{E}\left(Z\left(x_{1}, x_{2}\right) Z\left(x_{1}+\tau_{1}, x_{2}+\tau_{2}\right)\right) \\
& =\int_{\Re^{2}} z \tilde{z} \mathrm{p}_{Z\left(x_{1}, x_{2}\right), Z\left(x_{1}+\tau_{1}, x_{2}+\tau_{2}\right)}(z, \tilde{z}) \mathrm{d} z \mathrm{~d} \tilde{z},
\end{aligned}
$$

where $\mathbb{E}$ is the expectation operation, and $\mathrm{p}_{Z\left(x_{1}, x_{2}\right), Z\left(x_{1}+\tau_{1}, x_{2}+\tau_{2}\right)}$ is the second order marginal probability density function of the random field $(Z(\mathbf{x}, \theta), \mathbf{x} \in \mathfrak{D})$. For isotropic surfaces, the autocorrelation function is simplified as a $1 \mathrm{D}$ function as

$$
r_{Z}\left(\tau_{1}, \tau_{2}\right)=r_{Z}(\tau), \quad \forall\left\{\tau_{1}, \tau_{2}\right\} \in \mathfrak{R}^{2}: \tau=\sqrt{\tau_{1}^{2}+\tau_{2}^{2}}
$$


The random surface can be alternatively characterized by a PSD function defined as the Fourier transform of the correlation function, i.e.

$$
s_{Z}\left(\zeta_{1}, \zeta_{2}\right)=\int_{\Re^{2}} r_{Z}\left(\tau_{1}, \tau_{2}\right) \exp \left(-\mathrm{i}\left(\zeta_{1} \tau_{1}+\zeta_{2} \tau_{2}\right)\right) \mathrm{d} \tau_{1} \mathrm{~d} \tau_{2}
$$

where $\left\{\zeta_{1}, \zeta_{2}\right\}$ are the wave numbers and $\mathrm{i}^{2}=-1$. From the measurements of the surface height, e.g. using AFM, an approximation of the correlation function can be evaluated using Eq. (6) and the corresponding PSD function can be obtained using Eq. (8). In addition to this indirect method, the PSD function can be estimated directly from the measurements of the surface height as follows

$$
s_{Z}(\boldsymbol{\zeta})=\lim _{\chi \rightarrow \infty} \frac{1}{\chi^{2}}|\hat{z}(\boldsymbol{\zeta})|^{2},
$$

where $\chi \times \chi$ is the truncated size of the measurements, and $\hat{z}(\boldsymbol{\zeta})$ is the Fourier transform of the surface measurements $z(\mathbf{x})$,

$$
\hat{z}(\boldsymbol{\zeta})=\int_{\mathfrak{R}^{2}} \mathbb{1}_{[-\chi / 2, \chi / 2]^{2}}\{\mathbf{x}\} z(\mathbf{x}) \exp \left(-\mathrm{i} \zeta_{1} x_{1}-\mathrm{i} \zeta_{2} x_{2}\right) \mathrm{d} \mathbf{x}
$$

where $\mathbb{1}_{[-\chi / 2, \chi / 2]^{2}}\{\mathbf{x}\}$ is equal to 1 if $\mathbf{x} \in[-\chi / 2, \chi / 2]^{2}$ and equal to zero otherwise.

For the cases in which the involved contacting surfaces $\left\{\mathcal{S}_{1}, \mathcal{S}_{2}\right\}$ are statistically independent, the PSD function of the equivalent surface $\mathcal{S}$ can be constructed from their PSD functions $\left\{s_{Z_{(1)}}, s_{Z_{(2)}}\right\}$ as

$$
s_{Z}=s_{Z_{(1)}}+s_{Z_{(2)}}
$$

The normal height evolution $z(\mathbf{x}, \theta)$ corresponding to the surface realization $\mathcal{S}(\theta)$ can be generated using the PSD function $s_{Z}$ following the works of Shinozuka [44] and Poiron and Soize [45] as described in AppendixA. Fig. 3 illustrates a realization of contact surfaces using the PSD function that will be constructed in Section 4.

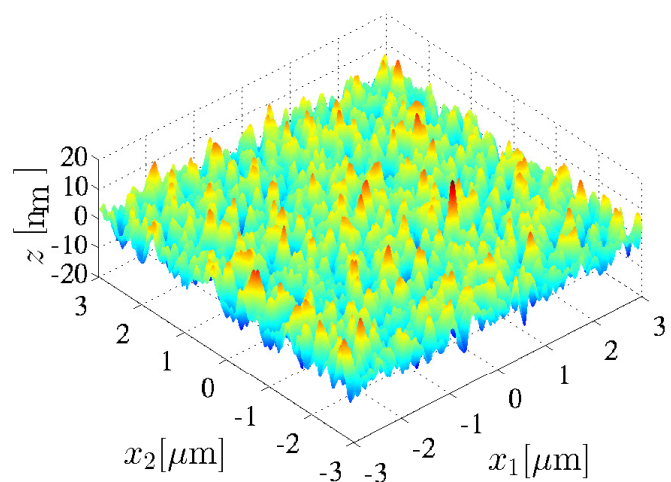

Figure 3: A generated surface sample using the PSD function constructed in Section 4.

\subsubsection{Numerical evaluation of the random apparent adhesive contact forces}

Once the rough surfaces $\mathcal{S}(\theta)$ are generated using the method described in Section 2.2.1, the semianalytical method developed in [14] is applied to evaluate the corresponding apparent adhesive contact forces $\bar{f}^{r}\left(\bar{d}^{r}, \theta\right)$ defined by Eq. (3) and illustrated in Fig. 2(c). In the following, the physical aspects of the capillary forces and vdW forces are described and two semi-analytical models used to evaluate the apparent adhesive contact force $\bar{f}^{r}$, in which the first one accounts for capillary forces and the second one accounts for both capillary and vdW forces, are summarized. 


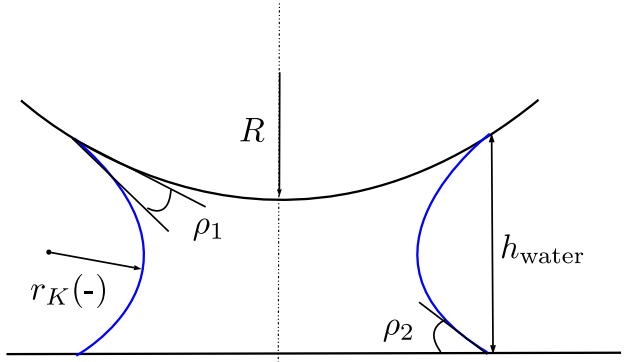

(a) Geometry of meniscus

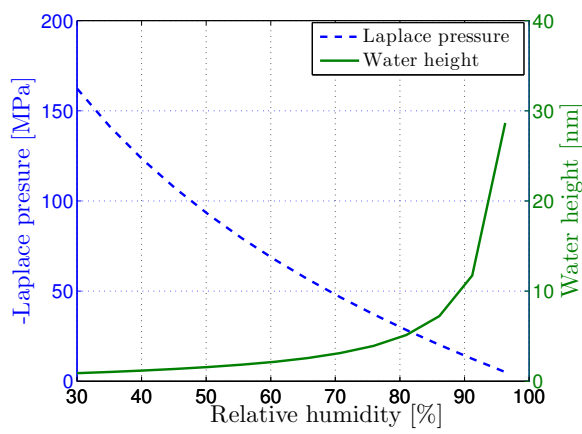

(b) Laplace pressure and water height

Figure 4: The configuration of condensing water. (a) Meniscus between a sphere and a half space. (b) The evolution of the Laplace pressure and of the water height in terms of the humidity level.

Capillary phenomena. The capillary forces result from the negative pressure inside the condensing water between two hydrophilic contacting surfaces, see Fig. 4(a). In terms of physics, during the separation process, the meniscus geometry varies depending on the competition between evaporation and condensation of water [10]. On the one hand, when the separation is rapid, the volume of meniscus is constant. On the other hand, if the separation is slow the radius of meniscus and its water pressure are constant. Between the two extreme cases, the adhesion energy of the latter is reduced by a factor of 2 [10]. In this paper, the assumption that the water pressure and the meniscus radius are constant is applied. That assumption respects the condition of the experiments reported in [5,9] which will be used for the experimental comparison in Section 5. With the constant pressure assumption, the pressure inside the meniscus is equal to the Laplace pressure, which is evaluated at a given relative humidity level $R H$ as $[10,46]$

$$
\Delta P=\frac{\gamma_{\mathrm{LG}}}{r_{K}}=\frac{\mathcal{R} T \ln R H}{V_{m}},
$$

where $\gamma_{\mathrm{LG}}$ is the liquid vapor energy, $V_{m}$ is the liquid molar volume, $\mathcal{R}$ is the universal gas constant, and $T$ is the absolute temperature. In case of water condensation $V_{m}=0.018 \mathrm{~L} / \mathrm{mol}$ and $\gamma_{\mathrm{LG}}=0.072 \mathrm{~N} / \mathrm{m}$ at $T=300 \mathrm{~K}$. The geometry of the menisci is characterized by the contact angles $\left\{\rho_{1}, \rho_{2}\right\}$, depending on the surfaces properties, and by the Kelvin radius $r_{K}$ given by [46]

$$
r_{K}=\frac{\gamma_{\mathrm{LG}} V_{m}}{\mathcal{R} T \ln R H}=\frac{0.53}{\ln R H}[\mathrm{~nm}]<0 .
$$

For the nano-scale rough surfaces, because the topology curvature is small, e.g. the radius of contacting sphere is much larger than the Kelvin radius see Fig. 4(a), the capillary range, defined as the maximum contact distance at which water can condense between two bodies, can be approximated by

$$
h_{C}=-2 r_{K}\left(\cos \left(\rho_{1}\right)+\cos \left(\rho_{2}\right)\right) \text {. }
$$

The capillary forces in the present work are modeled using Dugdale cohesive model, i.e. inside the range $h_{C}$ the pressure is constant and equal to the Laplace pressure, while outside this range the pressure vanishes. That model is consistent with the assumption of constant pressure [10].

The calculated Laplace pressures and condensing water heights for contact angles $\rho_{1}=\rho_{2}=0$ are illustrated in Fig. 4(b). It can be observed that the Laplace pressure is much smaller than the Young's modulus of hard materials such as poly-silicon, while the condensing water heights are comparable with the roughness of the typical MEMS surfaces [4, 5].

Remark: To simplify the numerical problem in the current work, the effects the absorbed surface layers [47], which are the water layers on the hydrophilic surfaces, are not accounted for. The work [47] showed that these absorbed surface layers are present in liquid state for humidity $R H \geq 60 \%$. 
Table 1: Dugdale model parameters of vdW forces.

\begin{tabular}{l|c|c} 
Environmental condition & Dry air $R H=0$ & Humid air $R H>0$ \\
\hline \hline$\omega_{\mathrm{vdW}}\left[\mathrm{mJ} / \mathrm{m}^{2}\right]$ & 167.1 & 87.4 \\
\hline$-\sigma_{\mathrm{vdW}}[\mathrm{MPa}]$ & 732 & 383 \\
\hline$h_{\mathrm{vdW}}[\mathrm{nm}]$ & 0.228 & 0.228
\end{tabular}

Van der Waals force. In this paper, we use Maugis theory [48] with Dugdale assumption to model the vdW adhesive elastic contact between a sphere and a flat surface.The model has been extended by Kim et al. [65] in the cases of non-contacting adhesive interactions between the sphere and the flat surface. The models implementation is detailed in [6].

The $\mathrm{vdW}$ interaction can be characterized by the adhesive energy per unit area, $\omega_{\mathrm{vdW}}$, given by

$$
\omega_{\mathrm{vdW}}=\frac{\mathfrak{H}}{16 \pi D_{0}^{2}}
$$

where $\mathfrak{H}$ is Hamaker constant, and $D_{0}$ is the equilibrium distance at which the force between two half space is zero and is given by $D_{0}=(2 / 15)^{1 / 6} r_{0}$ with $r_{0}$ the finite distance at which the inter-molecular potential is zero. For silicon, the Hamaker constant is $18.65 \times 10^{-20} \mathrm{~J}$ through dry air [49], corresponding to $R H \sim 0$, and $9.75 \times 10^{-20} \mathrm{~J}$ through water [49], which can be due to the menisci. In practice, the poly-silicon surfaces can be oxidized with thin layers $(2-3 \mathrm{~nm})$ of $\mathrm{SiO}_{2}$ [13], and the Hamaker value may be changed. However the change remains lower than one order of magnitude. For the sake of simplification, the Hamaker values reported in [49] are considered in this paper. For silicon, the distance $r_{0}=2.09 \AA$ leading to $D_{0}=1.49 \AA$. Using Dugdale assumption, the $\mathrm{vdW}$ stress, $\sigma_{\mathrm{vdW}}$, is constant for the interaction distances smaller than the critical separation $h_{\mathrm{vdW}}$, and vanishes outside this range. The energy balance leads to the relationship $\omega_{\mathrm{vdW}}=-\sigma_{\mathrm{vdW}} \times h_{\mathrm{vdW}}$. For silicon, the range $h_{\mathrm{vdW}}$ can be deduced as $h_{\mathrm{vdW}}=0.97 \times 2^{1 / 6} r_{0}=2.28 \AA[7]$. The modeling parameters of vdW interaction are reported in Tab. 1 . In comparison with the capillary forces range $h_{\mathrm{C}}(\sim \mathrm{nm})$, the $\mathrm{vdW}$ force range $h_{\mathrm{vdW}}$ is much shorter.

Numerical model to evaluate the apparent adhesive contact forces. When two rough surface bodies interact, while the vdW and capillary adhesive forces pull them together, the elastic forces resulting from the bodies deformation tend to separate them. Those forces contribute to the apparent adhesive contact forces and must be accounted for in the numerical model. We develop two numerical models, the first one accounts only for the capillary forces as the adhesive source, and the second one accounts for both capillary and vdW forces.

In cases of capillary contact problems on (relative) hard materials, the repulsive contact force results from the elastic stress concentrating at the highest asperities while the adhesive contact force results from adhesive pressures acting on the zones surrounding these asperities, see Fig. 1(b). In this kind of problems, because the elastic stress is much larger than the Laplace pressure, at equilibrium the water condensing area is much wider than the physical contact areas between asperities. For those cases, the adhesive part and the repulsive part of the contact force can be evaluated separately [28], and the deformation of asperities is caused mainly by the elastic stress [14].

To evaluate the contact forces for the capillary contact problems on hard materials, the semi-analytical method developed in [14] can be applied. That method is based on the assumption of the separation between the adhesive and the repulsive forces, and follows the 5 steps illustrated in Fig. 5:

(i) Identifying the physical contacting asperities using the topology of the contacting surfaces;

(ii) Fitting the contacting asperities profile by a sphere, see e.g. [14, 50, 51], as illustrated in Fig. 6;

(iii) Evaluating the asperity contact forces and the physical contacting areas by applying the analytical Hertz contact theory [26] on the sphere-fitted asperities; 


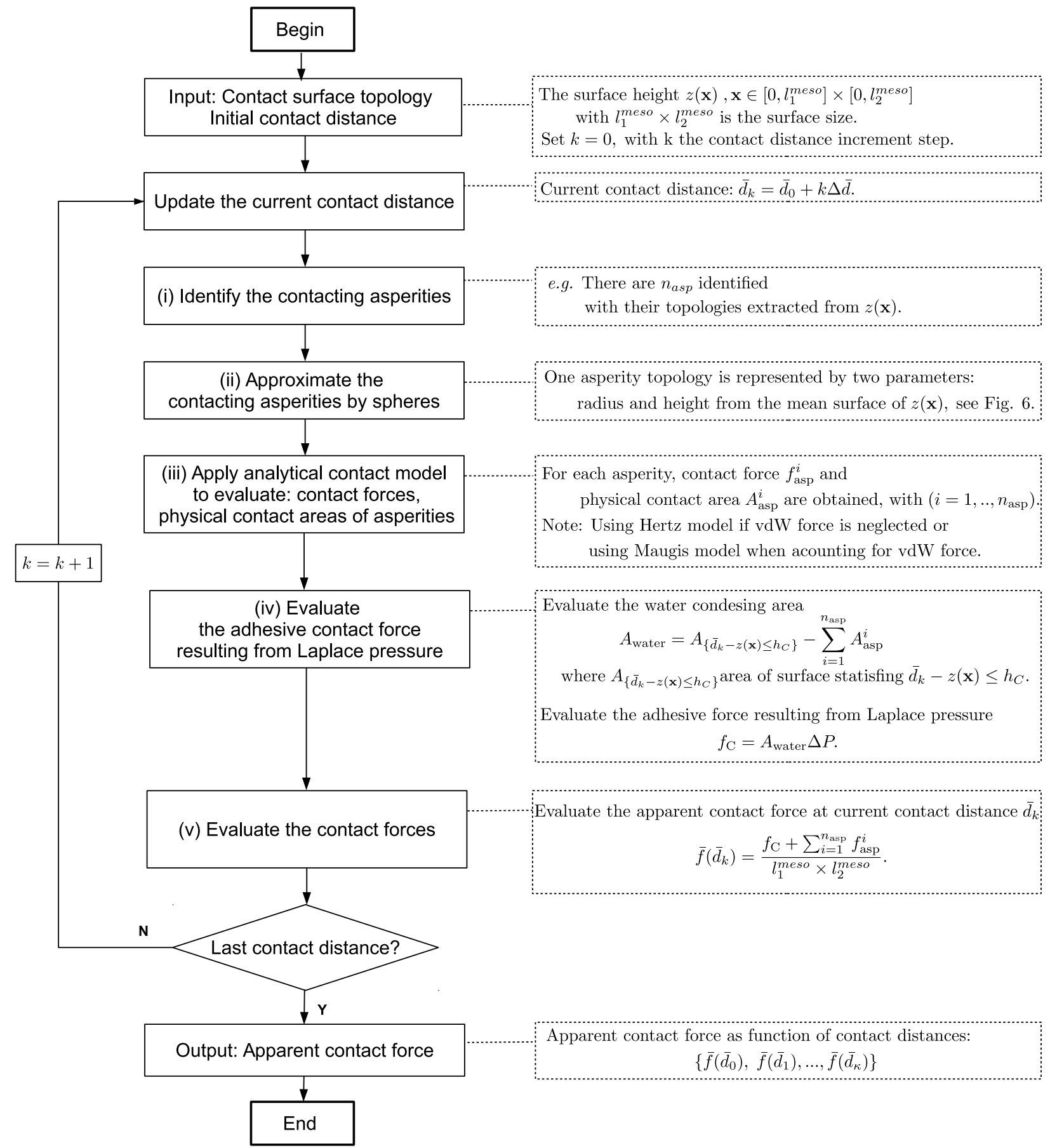

Figure 5: The algorithm of the semi-analytical contact models. 


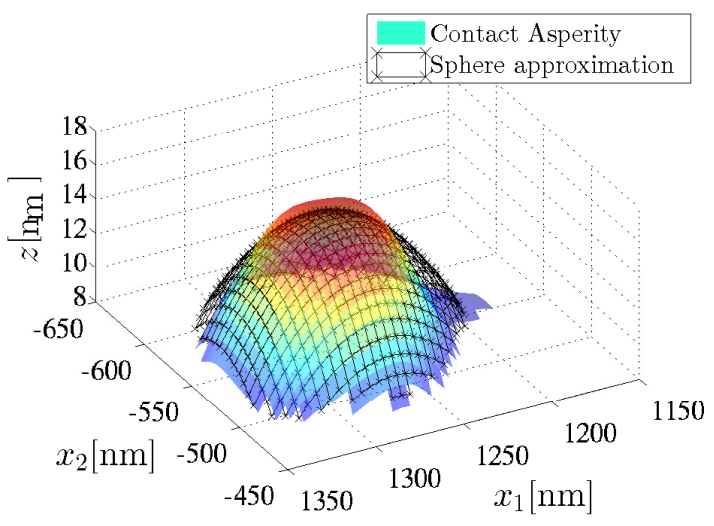

(a)

Figure 6: An identified contacting asperity extracted from surface topology, see Fig. 3, and its spherical approximation.

(iv) Evaluating the adhesive contact force: first, the area on which the water condenses is identified as the difference between the area of parts of surface topology with interaction distances smaller than the capillary range $h_{C}$, and the sum of physical asperity contacting areas calculated from step (iii); then the adhesive contact forces are calculated by multiplying the water condensing area with the Laplace pressure evaluated by Eq. (12);

(v) Computing the contact forces as the sum of (1) the asperity contact forces, resulting from the elastic interactions and obtained by step (iii), and (2) the adhesive contact force, resulting from Laplace pressure and evaluated from step (iv).

Inside step (iv), the area of condensing water is calculated in a global way instead of considering each asperity separately. In that way, the saturation effect in which some water menisci can merge together at the high humidity levels [4] is accounted for as described in [14]. The apparent contact forces are required to be evaluated for a discrete set of contact distances $\left\{\bar{d}^{0}, \bar{d}^{0}+\Delta \bar{d}, \ldots, \bar{d}^{0}+\kappa \Delta \bar{d}\right\}$ with $\Delta \bar{d}$ the sampling distance. Therefore, that process is repeated for $\kappa+1$ times in order to construct an apparent contact force vs. distance curve, see Fig. 5 for its algorithm sketch.

The model can be extended to account for the vdW forces. Because the vdW force range $h_{\mathrm{vdW}}=0.228[\mathrm{~nm}]$ is much smaller than the capillary force range $h_{\mathrm{C}}$ and comparable with the penetration of asperities, the contact forces resulting from vdW stress and elastic deformation stress are evaluated locally at each contacting asperity using Maugis adhesive contact model [48]. In that way, both elastic stress and vdW stress are accounted for the deformation of asperities. With that assumption the steps (iii) and (v) are modified to account for the vdW force as:

(iii) Evaluating the asperity contact forces and physical contact areas resulting from vdW forces and elastic interaction by applying the analytical Maugis adhesive contact theory [48], extended by Kim et al. [65] for the non-contacting cases, on the sphere-fitted asperities with the input parameters $h_{\mathrm{vdW}}, \sigma_{\mathrm{vdW}}$, and $\omega_{\mathrm{vdW}}$ given in Tab. 1 ;

(v) Computing contact forces as the sum of (1) the asperity contact forces, resulting from vdW forces and elastic interaction obtained by step (iii), and (2) the capillary adhesive contact force, resulting from Laplace pressure and evaluated from step (iv) as discussed previously,

while the other steps are preserved.

Remarks: (i) These methods are performed based on the generated (or measured) surfaces with the size of interest, e.g. the size of sub-contact domain $l_{1}^{\text {meso }} \times l_{2}^{\text {meso }}$. They are thus able to account for the size effects required in this work. (ii) The Hertz model and Maugis model applied on the sphere-fitted asperities 
are evaluated using the equivalent Young's modulus $E_{\mathrm{eq}}=\left(\left(1-\nu_{1}^{2}\right) / E_{1}+\left(1-\nu_{2}^{2}\right) / E_{2}\right)^{-1}$ where $E_{1}, E_{2}, \nu_{1}$, $\nu_{2}$ are the Young's modulus and Poisson ratio of the two contacting bodies. (iii) As both vdW stress and Laplace pressure are much smaller than the ultimate strength of poly-silicon, and the contacting surfaces have a small roughness $(\sim 3.5 \mathrm{~nm})$, in this paper the irreversible behaviors are neglected when evaluating the asperity interaction. For the cases when irreversible deformations become non-negligible [52], the applied asperity contact models, Hertz and Maugis models, should be replaced, e.g. by the ones developed by L. Kogut and I. Etsion [53,54], or by the one developed by L. Wu et al. [8] in the case of cyclic load.

\subsection{Stochastic FE model for adhesive contact involved structures using the random apparent contact forces}

At the upper scale, for an elastic problem, using the weak formulation stated in Eq. (1) with the upper scale contact forces given by Eq. (5), the structural behaviors can be identified by solving the FE model equations

$$
[K] \mathbf{u}(\theta)=\mathbf{f}^{\mathrm{c}}(\mathbf{u}, \theta)+\mathbf{f}^{\mathrm{a}},
$$

where $[K]$ is the stiffness matrix, $\mathbf{u}$ is the nodal displacements vector, $\mathbf{f}^{\mathbf{a}}$ is the equivalent nodal external forces vector resulting from the applied forces, and $\mathbf{f}^{\mathrm{c}}$ is the equivalent nodal contact forces vector resulting from the homogenized contact forces defined in Eq. (5) and evaluated using the semi-analytical contact models developed in Section 2.2.2. The problem is non-linear because the nodal forces $\mathbf{f}^{\mathrm{c}}$ depend on the actual configuration $\mathbf{u}$.

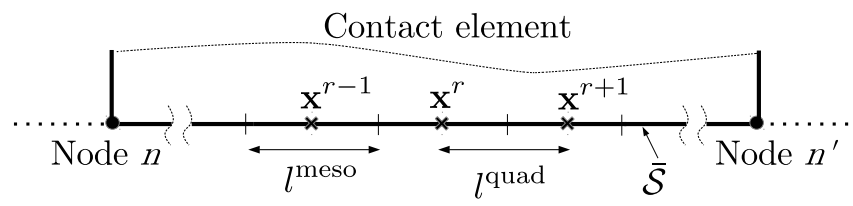

Figure 7: 1D contact rectangular element with equally spaced integral points $\mathbf{x}^{r}$ used to evaluate the nodal contact forces.

In order to evaluate the nodal contact forces $\mathbf{f}^{\mathrm{c}}$ corresponding to a realization of random surface $\mathcal{S}(\theta)$, this work has recourse to the rectangular type quadrature rule in which the integral points are equally spaced with a distance $l^{\text {quad }}$ chosen to be equal to the meso-scale length, $l^{\text {quad }}=l^{\text {meso }}$, see e.g. Fig. 7 . To this end, the surfaces $\mathcal{S}(\theta)$ and $\overline{\mathcal{S}}$ are decomposed into $N_{p}$ non-overlapping surfaces $\mathcal{S}^{r}(\theta)$ and $\overline{\mathcal{S}}^{r}$ respectively, with $r=1, \ldots, N_{p}$ and with size $l_{1}^{\text {meso }} \times l_{2}^{\text {meso }}$. The central point of the surfaces $\overline{\mathcal{S}}^{r}$ is considered as the integral point $\mathbf{x}^{r}$. For each sub-contact domain, the apparent adhesive contact force $\bar{f}^{r}(\theta)$, defined by Eq. (3), is evaluated using the semi-analytical contact models developed in Section 2.2.2. The nodal forces, $\mathbf{f}^{\mathrm{c}, n}$, at the node $n$ belonging to the mean surfaces $\overline{\mathcal{S}}$ are computed by applying the FE discretization on the homogenized contact force defined in Eq. (5), leading to

$$
\mathbf{f}^{\mathrm{c}, n}(\mathbf{u}, \theta)=\sum_{r=1}^{N_{p}} \bar{f}^{r}\left(\bar{d}^{r}(\mathbf{u}), \theta\right) \Psi^{\mathrm{FE}, n}\left(\mathbf{x}^{r}\right) \mathbf{n}_{\overline{\mathcal{S}}}\left(\mathbf{x}^{r}\right) A_{\overline{\mathcal{S}}^{r}}
$$

where $\Psi^{\mathrm{FE}, n}$ is the shape function of the corresponding node, $\bar{d}^{r}$ is evaluated as the normal distance from the integral point $\mathbf{x}^{r}$ to the encountering plane at the actual configuration defined by $\mathbf{u}$, and $\mathbf{n}_{\overline{\mathcal{S}}}$ is the normal vector of mean surface $\overline{\mathcal{S}}$.

The probabilistic structural behaviors are obtained by solving the set of Eqs. (16) using the input of the random apparent contact forces which are either explicitly evaluated, as previously discussed, or generated through a constructed stochastic model, see next Section.

Remarks: (i) For conventional multiscale models in which the apparent contact force is deterministic, the distance between two adjacent integral points is much bigger than the meso-scale length, $l^{\text {quad }} \gg l^{\text {meso }}$. For the present case, the apparent contact forces are non-deterministic, see Fig. 2(c), in order to account for the size effect on their scatter, the distance between two adjacent integral points, is chosen to be equal to the 
meso-scale length, $l^{\text {quad }}=l^{\text {meso }}$. This choice will be justified in Section 4.4.1 where the spatial correlation will be discussed. This is also the reason of applying the quadrature rule with equally spaced points when evaluating the nodal contact forces. (ii) The probabilistic multiscale framework is developed for the following cases: the mean surfaces of the contacting rough surfaces are non-parallel, however at the meso-scale size $l_{1}^{\text {meso }} \times l_{2}^{\text {meso }}$, the non-parallel degree is much smaller than the ratio between the considered roughness and the meso-scale length. In this paper, that assumption is valid when considering the application to MEMS cantilever beams, see analyses in Section 4.2. In the following, two scenari in which that assumption is not valid are discussed. (a) When the non-parallel degree at the meso-scale is comparable with or larger than the ratio between the considered roughness and the meso-scale length, the multiscale method should be modified to account for the lack of parallelism. For instance, when evaluating the meso-scale apparent contact forces Eq. (3), in addition to the contact distance $\bar{d}^{r}$ and the uncertainty parameter $\theta$, other parameters such as the surfaces interaction angle can be added. In this case, the meso-scale contact forces are evaluated for non-parallel mean surfaces according to the values of these additional parameters. (b) When the structural contact geometries are not between two surfaces anymore, e.g. between an edge of a triangular prism and a surface, the multiscale approach is modified by changing the notion of the mean surface $\bar{S}$ by the successive interactions of two non-parallel surfaces couples. For the sake of simplification when evaluating the apparent contact forces and when developing the stochastic method, we assume that the surfaces are nominally parallel, the cases (a) and (b) being beyond the scope of this paper.

\section{Effective probabilistic representation of the random apparent adhesive contact forces}

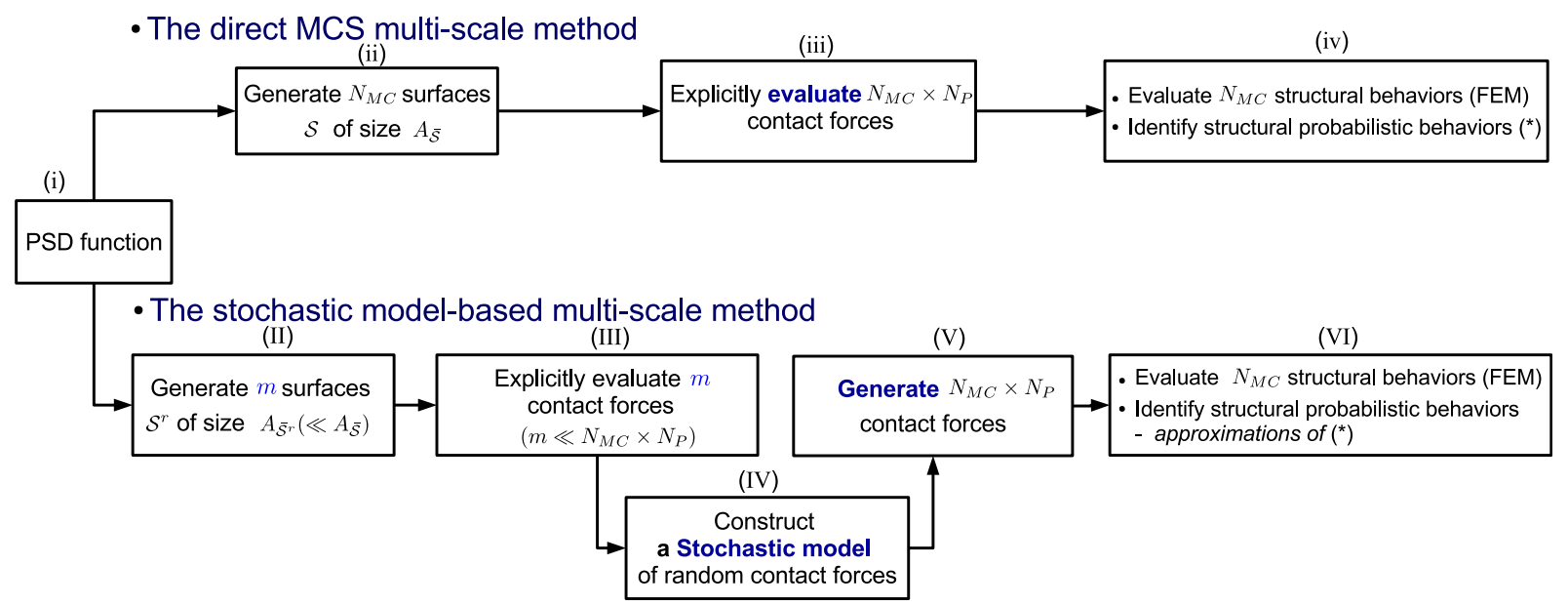

Figure 8: The two uncertainty propagation methods used to evaluate the probabilistic behaviors of structures involving adhesive contacts: the direct MCS multiscale method and the stochastic model-based multiscale method.

In order to quantify the uncertainties of structural behaviors involving adhesive contacts, two uncertainty propagation methods, the direct MCS method and the stochastic model-based method, illustrated in Fig. 8, are considered to be coupled with the multiscale model. The purpose of developing the latter is to overcome the computational inefficiency nature of the direct MCS method. The direct MCS method explicitly evaluates the sub-contact domain random apparent adhesive contact forces, while the stochastic model-based method constructs a stochastic model which is then used to generate realizations of these apparent contact forces.

Direct MCS multiscale method. The direct MCS multiscale method follows 4 steps, see Fig. 8:

(i) From the measurements of contacting surfaces, e.g. using AFM, the PSD function can be estimated, see Section 2.2.1; 
(ii) $N_{M C}$ surfaces with the size of the contacting surface, $\mathcal{S}^{(k)}$ with $k=1, \ldots, N_{M C}\left(\mathcal{S}^{(k)}\right.$ the simplified form of its detailed notation $\mathcal{S}\left(\theta^{k}\right)$ ), are generated using the estimated PSD, see Section 2.2.1;

(iii) Each generated surface $\mathcal{S}^{(k)}$ is discretized into $N_{p}$ non-overlapping surfaces of size $l_{1}^{\text {meso }} \times l_{2}^{\text {meso }}$, denoted by $\mathcal{S}^{(k), r}$ with $r=1, \ldots, N_{p}$, and the corresponding apparent contact forces, $\bar{f}^{(k), r}$ with $r=1, \ldots, N_{p}$, are evaluated using the methodology described in Section 2.2.2;

(iv) The structural behavior corresponding to the $k$ th generated surface $\mathcal{S}^{(k)}$ is computed by integrating the $N_{p}$ apparent contact forces, $\bar{f}^{(k), r}$ with $r=1, \ldots, N_{p}$, into the FE model of the studied structure using Eq. (17). To avoid the numerical obstacles, the evaluated contact forces are curve fitted with a $C^{1}$ function derived from Morse potential, as described in Section 3.1, for their evaluation in the FE model. This step is repeated $N_{M C}$ times corresponding to the $N_{M C}$ generated surfaces $\mathcal{S}^{(k)}$. From the set of $N_{M C}$ evaluated structural behaviors we can identify the structural properties distribution.

The point of computational resources complexity is the step (iii) in which there are $N_{M C} \times N_{p}$ apparent contact forces required to be evaluated.

Stochastic model-based multiscale method. To reduce the computational cost, we construct a stochastic model that approximates the distribution of the random apparent contact force and is used to generate the contact force realizations. The input required to develop the stochastic model is $m$ apparent contact forces explicitly evaluated from the direct MCS method. The cost of computational resources is reduced thanks to the fact that $m \ll N_{M C} \times N_{p}$. We remark that the stochastic model-based multiscale method also performs MCS however in an indirect way through the stochastic model of apparent contact forces. The 5 steps of the method are illustrated in Fig. 8. Among them, only the steps (IV,V), the construction and the use of the stochastic model of the random apparent contact forces, are not discussed yet, and are the main object of this section.

To construct the stochastic model, the apparent contact force $\bar{f}^{r}(\bar{d})$, Eq. (3), is first parametrized and represented by a vector $\mathbf{v}$ to be defined. As the apparent contact forces are uncertain, their representative vectors $\mathbf{v}$ are treated here as a random vector, denoted by $\mathbf{V}$. The randomness of the parameters vectors $\mathbf{V}$ is then represented by a stochastic model using the gPCE. With the constructed stochastic model, $N_{M C} \times N_{p}$ apparent contact forces can then be generated efficiently.

In the developments of this section, we discuss the main ingredients of the stochastic model-based multiscale method for adhesive problems:

(i) The parametrization of the apparent contact forces by the parameters vectors $\mathbf{v}$;

(ii) The construction of a gPCE model for the random parameters vectors $\mathbf{V}$.

\subsection{Parametrization of the apparent adhesive contact forces}

The calculated apparent adhesive contact forces are first characterized by a set of physical parameters and then fitted by an analytical function derived from the Morse potential.

\subsubsection{Characterization of the apparent adhesive contact forces}

In the context of adhesive problems, there are 4 key features characterizing the adhesive contact force $\bar{f}$ (the superscript " $r "$ is dropped for simplicity), Eq. (3), see Fig. 9, which are listed here below.

(i) The maximum pull-out adhesive force $\bar{f}_{\max }$ defined by

$$
\bar{f}_{\max }=\max _{\bar{d}}\{-\bar{f}(\bar{d})\}>0
$$

(ii) The distance $\bar{d}_{\max }$ at which the apparent contact force reaches its maximum adhesive force

$$
\bar{d}_{\max }=\arg \max _{\bar{d}}\{-\bar{f}(\bar{d})\} ;
$$



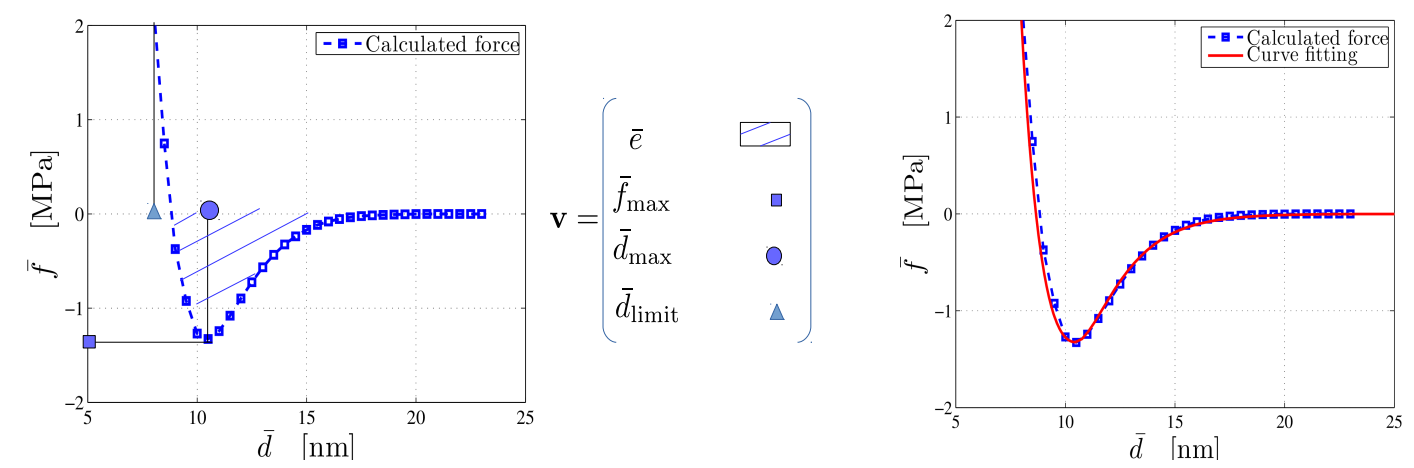

Figure 9: Curve fitting of a typical apparent adhesive contact force. Left figure: An explicitly evaluated apparent contact force, see Section 2.2, and its parameters. Right figure: The evaluated apparent contact force represented by the analytical function, Eq. (22).

(iii) The apparent adhesive energy, $\bar{e}$, defined by

$$
\bar{e}=-\int_{\mathfrak{R}} \mathbb{1}_{\leq 0}\{\bar{f}(\bar{d})\} \bar{f}(\bar{d}) \mathrm{d} \bar{d}>0
$$

where $\mathbb{1}_{\leq 0}\{\cdot\}=1$ if $\cdot \leq 0$ and zero otherwise;

(iv) The threshold distance $\bar{d}_{\text {limit }}$ at which the apparent contact force reaches a certain limit positive force $\bar{f}_{\text {limit }}$ defined as the maximum considered compressive force of interest. Beyond this limit force, the compressive behavior dominates the adhesive behavior, and therefore is not in the scope of an adhesive contact study.

These 4 key features of the adhesive contact force are represented by the parameters vector

$$
\mathbf{v}=\left[\bar{e}, \bar{f}_{\max }, \bar{d}_{\max }, \bar{d}_{\text {limit }}\right]^{\mathrm{T}} .
$$

\subsubsection{Curve fitting of the apparent adhesive contact forces using analytical function}

The following function is considered to fit the apparent adhesive contact forces

$$
\phi(\bar{d})=\left\{\begin{array}{l}
\bar{f}_{\max }\left(\mathrm{e}^{-2 a_{\mathrm{right}}\left(\bar{d}-\bar{d}_{\mathrm{max}}\right)}-2 \mathrm{e}^{-a_{\mathrm{right}}\left(\bar{d}-\bar{d}_{\mathrm{max}}\right)}\right) \quad \text { for } \quad \bar{d} \geq \bar{d}_{\mathrm{max}} \\
\bar{f}_{\max }\left(\mathrm{e}^{-2 a_{\mathrm{left}}\left(\bar{d}-\bar{d}_{\max }\right)}-2 \mathrm{e}^{-a_{\mathrm{left}}\left(\bar{d}-\bar{d}_{\max }\right)}\right) \quad \text { for } \quad \bar{d}<\bar{d}_{\max }
\end{array}\right.
$$

In fact, the fitting analytical function is derived from the well-known Morse potential ${ }^{2}$. By separating the two wings with the different coefficients $\left(a_{\text {right }}\right.$ and $\left.a_{\text {left }}\right)$, the proposed function has 4 fitting coefficients, one more coefficient than the original Morse formulation. This fitting function reaches its maximum adhesive force $\bar{f}_{\text {max }}$ at a distance $\bar{d}_{\text {max }}$. The other coefficients of the fitting function $\left\{a_{\text {right }}, a_{\text {left }}\right\}$ are identified by solving the following equations

$$
\begin{aligned}
& \bar{f}_{\text {max }}\left(\mathrm{e}^{-2 a_{\text {left }}\left(\bar{d}_{\text {limit }}-\bar{d}_{\text {max }}\right)}-2 \mathrm{e}^{-a_{\text {left }}\left(\bar{d}_{\text {limit }}-\bar{d}_{\text {max }}\right)}\right)=\bar{f}_{\text {limit }} ; \\
& -\int_{\mathfrak{R}} \mathbb{1}_{\leq 0}\left\{\phi\left(\bar{d} ; a_{\text {right }}, a_{\text {left }}\right)\right\} \phi\left(\bar{d} ; a_{\text {right }}, a_{\text {left }}\right) \mathrm{d} \bar{d}=\bar{e} .
\end{aligned}
$$

One can thus obtain the physical parameters vector, Eq. (21), from a given apparent contact force and also can reconstruct that apparent contact force knowing its physical parameters vector using Eq. (22). In other words, an apparent contact force can be represented by its parameters vector $\mathbf{v}$, Eq. (21).

\footnotetext{
${ }^{2}$ We remark that the Morse potential, used to fit energy functions, and its derivative, used to fit distance-force curves, have the same characteristics as they are composed by the exponential functions. Thus, for simplicity, the analytical function in Eq. (22) uses Morse potentials directly instead of its derivative to fit the adhesive contact forces.
} 


\subsection{Construction the generator of the random apparent adhesive contact forces using the gPCE}

The main idea of the present work is to construct a stochastic model using the gPCE to generate the realizations of parameters vectors $\mathbf{v}$ from which the corresponding apparent adhesive contact forces are obtained using the adhesive contact functions defined in Eq. (22). The stochastic model is constructed based on a set of $m$ explicitly evaluated apparent contact forces, from which the set of $m$ corresponding physical parameters vectors $\left\{\mathbf{v}^{(1)}, \ldots, \mathbf{v}^{(m)}\right\}$-samples of the random vector $\mathbf{V}$ - is obtained using the parametrization process described in Section 3.1.

As it will be shown in Section 4, the spatial correlation between two adjacent integral points of the random apparent contact forces $\bar{f}^{r}$ is negligible. It is due to the two facts: (i) the distance between two integral points $l^{\text {quad }}=l^{\text {meso }}$, see Fig. (7), is longer than the correlation length $l^{\mathrm{m}}$ of the contacting surface as discussed in Section 2, and (ii) the contact happens only at the highest asperities with a physical contact area of $\mathcal{O}(1 \%)$ of the apparent area. Therefore, the stochastic model developed in the following does not take the spatial correlation into account. For the cases of non-negligible spatial correlation, the model can be extended, e.g. by coupling a Karhunen-Loève expansion with the PCE as developed in [31, 32, 36].

In the considered problem, the construction of the stochastic model must deal with two challenges: (i) the stochastic model must respect the physical bounds of the modeled random variables i.e. their generated samples are preconditioned by those bounds; and (ii) the effect of curse of dimensionality [37], i.e. the number of orthogonal polynomials in a gPCE model which increases exponentially with the dimensionality, must be minimized. These challenges are accounted for in Section 3.2.1, before developing the stochastic model in Section 3.2.2.

\subsubsection{Input data processing}

\begin{tabular}{|c|c|c|c|}
\hline & $\begin{array}{l}\text { (i) Account for } \\
\text { physical bounds }\end{array}$ & \multicolumn{2}{|c|}{ (ii) Dimension reduction } \\
\hline \multirow{2}{*}{\begin{tabular}{|c|} 
Random vector $\mathbf{V}$ \\
samples: $\left\{\mathbf{v}^{(1)}, \ldots, \mathbf{v}^{(m)}\right\}$
\end{tabular}} & & \multirow{2}{*}{$\begin{array}{c}\text { Random vector } \mathbf{Q} \\
\text { samples: }\left\{\mathbf{q}^{(1)}, \ldots, \mathbf{q}^{(m)}\right\}\end{array}$} & Random vector $\widetilde{\mathbf{H}}$ \\
\hline & & & samples: $\left\{\tilde{\boldsymbol{\eta}}^{(1)}\right.$, \\
\hline
\end{tabular}

Figure 10: The two data processing procedures, treatment of physical bounds, and dimension reduction.

In this section, the two data processing procedures illustrated in Fig. 10 are described. The first procedure introduces the random vector $\mathbf{Q}$ which accounts for the physical bounds applied on $\mathbf{V}$. In the second procedure, a statistical dimension reduction is applied on the random vector $\mathbf{Q}$ and the reduced dimension random vector $\widetilde{\mathbf{H}}$ is obtained. These two procedures are accomplished in such a way that their inverse processes, $\widetilde{\mathbf{H}} \rightarrow \mathbf{Q} \rightarrow \mathbf{V}$ exist. As a result, the stochastic model for the random vector $\mathbf{V}$ is constructed indirectly through the random vector $\widetilde{\mathbf{H}}$ in Section 3.2.2.

Introduction of physical bounds. The random vector $\mathbf{V}$ is constrained by the physical conditions:

(i) The 2 variables $\left\{\bar{e}, \bar{f}_{\max }\right\}$ are non-negative; and

(ii) The distance $\bar{d}_{\max }$ at which the apparent contact force reaches its maximum adhesive force is larger than the threshold distance $\bar{d}_{\text {limit }}$, see Fig. 9 .

These two constraints have to be taken into account in the way of constructing the stochastic model to ensure consistency. Toward this end, we introduce a standardized random vector $\mathbf{Q}$ valued in $\mathfrak{R}^{4}$ for which its samples $\left\{\mathbf{q}^{(1)}, \ldots, \mathbf{q}^{(m)}\right\}$ are evaluated from the parameters vectors $\left\{\mathbf{v}^{(1)}, \ldots, \mathbf{v}^{(m)}\right\}$ as

$$
\mathbf{q}^{(k)}=\left[\frac{\log \left(\bar{e}^{(k)}\right)}{\sigma_{\log (\bar{e})}}, \frac{\log \left(\bar{f}_{\max }^{(k)}\right)}{\sigma_{\log \left(\bar{f}_{\max }\right)}}, \frac{\bar{d}_{\max }^{(k)}}{\sigma_{\bar{d}_{\max }}}, \frac{\log \left(\bar{d}_{\max }^{(k)}-\bar{d}_{\text {limit }}^{(k)}\right)}{\sigma_{\log \left(\bar{d}_{\max }-\bar{d}_{\text {limit }}\right)}}\right]^{\mathrm{T}}, \quad \text { with } k=1, \ldots, m,
$$

where $\sigma$. is the standard derivation of the random variable $\cdot$ evaluated from $m$ samples $\left\{\mathbf{v}^{(1)}, \ldots, \mathbf{v}^{(m)}\right\}$, e.g.

$$
\sigma_{\log (\bar{e})} \approx\left[\frac{\sum_{k=1}^{m}\left(\log \left(\bar{e}^{(k)}\right)-\mathbb{E}\{\log (\bar{e})\}\right)^{2}}{m-1}\right]^{1 / 2}
$$


with

$$
\mathbb{E}\{\log (\bar{e})\} \approx \frac{\sum_{k=1}^{m} \log \left(\bar{e}^{(k)}\right)}{m} .
$$

The transformation in Eq. (24) is bijective and its inverse is given by

$$
\mathbf{v}=\left[\exp \left(q_{1} \sigma_{\log (\bar{e})}\right), \exp \left(q_{2} \sigma_{\log \left(\bar{f}_{\max }\right)}\right), q_{3} \sigma_{\bar{d}_{\max }}, q_{3} \sigma_{\bar{d}_{\max }}-\exp \left(q_{4} \sigma_{\log \left(\bar{d}_{\max }-\bar{d}_{\text {limit }}\right)}\right)\right]^{\mathrm{T}} .
$$

Using the stochastic model that is developed in Section 3.2.2, one can generate realizations q, from which the corresponding realizations $\mathbf{v}$ can be evaluated using Eq. (27), while respecting their physical bounds. In Eq. (27), the superscript index ${ }^{(k)}$ has been neglected in order to conserve its generality which is useful for the development of the generator of the apparent contact forces discussed in Section 3.2.2.

Linear dimension reduction. To reduce the effect of curse of dimensionality, a linear dimension reduction process is applied before the gPCE to represent the randomness of the reduced dimension random variables. The idea of the linear dimension reduction is to seek for a few orthogonal linear combinations of the original variables with the largest variances. In this work, the linear dimension reduction is performed using the principal component analysis of the covariance matrix $[55,56,57]$.

Using the input data of $m$ vectors $\left\{\mathbf{q}^{(1)}, \ldots, \mathbf{q}^{(m)}\right\}$ evaluated from Eq. (24) one can estimate their mean vector $\overline{\mathbf{q}}$ and their covariance matrix $\left[C_{\mathbf{Q}}\right]$ as

$$
\overline{\mathbf{q}} \approx \frac{\sum_{k=1}^{m} \mathbf{q}^{(k)}}{m}, \quad\left[C_{\mathbf{Q}}\right] \approx \frac{\sum_{k=1}^{m}\left(\mathbf{q}^{(k)}-\overline{\mathbf{q}}\right)\left(\mathbf{q}^{(k)}-\overline{\mathbf{q}}\right)^{\mathrm{T}}}{m-1} .
$$

Applying the principal component transformation, we deduce the random vector $\mathbf{H}$ with its realization $\boldsymbol{\eta}$ evaluated as

$$
\boldsymbol{\eta}^{\mathrm{T}}=(\mathbf{q}-\overline{\mathbf{q}})^{\mathrm{T}}[A][\Lambda]^{-1 / 2},
$$

where $[A]=\left[\mathbf{a}_{1}, \ldots, \mathbf{a}_{4}\right]$ is a matrix of the 4 eigenvectors of the matrix $\left[C_{\mathbf{Q}}\right]$ satisfying $[A][A]^{\mathrm{T}}=I_{4}$ with $I_{4}$ the 4 -dimensional unit vector, and $[\Lambda]=\operatorname{diag}(\boldsymbol{\lambda})$ is a diagonal matrix of the 4 corresponding ordered eigenvalues $\lambda_{1} \geq \ldots \geq \lambda_{4} \geq 0$. The random vector $\mathbf{H}$ is characterized by a zero mean and identity covariance matrix, and its components are uncorrelated in the sense of covariance ${ }^{3}$. The 4-dimensional random vector Q can be represented by the $N_{g}$-dimensional $\left(N_{g} \leq 4\right)$ random vector $\widetilde{\mathbf{H}}$ with its realization $\widetilde{\boldsymbol{\eta}}$ defined by the first $N_{g}$ components of vector $\boldsymbol{\eta}$,

$$
\widetilde{\boldsymbol{\eta}}=\left\{\eta_{1}, \ldots, \eta_{N_{g}}\right\},
$$

where $N_{g}$ is the reduced dimension. That dimensional reduction is obtained by the following approximation,

$$
q_{i} \simeq \bar{q}_{i}+\sum_{j=1}^{N_{g}} \sqrt{\lambda_{j}} A_{i j} \eta_{j}, \quad \text { for } i=1, \ldots, 4 .
$$

The superscript " $\sim$ is added in the notations $\widetilde{\mathbf{H}}(\widetilde{\boldsymbol{\eta}})$ in order to indicate the reduced dimension quantities, and to differ them from the original quantities $\mathbf{H}(\boldsymbol{\eta})$. The number of reduced dimensions $N_{g}$ can be chosen in order to verify the condition

$$
\operatorname{Err}^{\mathrm{DR}}\left(N_{g}\right)=\frac{\sum_{i=N_{g}+1}^{4} \lambda_{i}}{\sum_{i=1}^{4} \lambda_{i}}<\epsilon,
$$

where $\epsilon \ll 1$ is the admitted error.

\footnotetext{
${ }^{3}$ We remark that uncorrelated random variables are not necessarily independent
} 


\subsubsection{Stochastic model}

Because the sequential transformation $\widetilde{\mathbf{H}} \stackrel{\text { Eq. (31) }}{\longrightarrow} \mathbf{Q} \stackrel{\text { Eq. (27) }}{\longrightarrow} \mathbf{V}$ exists, the reduced dimension random vector $\widetilde{\mathbf{H}}$ can represent the random vector $\mathbf{V}$ of apparent contact force parameters. In this section, a stochastic model is constructed in order to generate the realizations of the random vector $\widetilde{\mathbf{H}}$ using its $m$ explicitly evaluated samples $\left\{\tilde{\boldsymbol{\eta}}^{(1)}, \ldots, \tilde{\boldsymbol{\eta}}^{(m)}\right\}$, obtained using Eqs. (29, 30), as input data. The stochastic model developed in this paper is a truncated $N_{d}$ order gPCE mapping the random vector $\boldsymbol{\Xi}$, uniformly distributed in the $N_{g}$-dimensional unit cube $[0,1]^{N_{g}}$, to the random vector $\widetilde{\mathbf{H}}^{\text {PC }}$, which is enforced to be an approximation in terms of distribution of the objective random vector $\widetilde{\mathbf{H}}$. That truncated gPCE is formulated as

$$
\underbrace{\widetilde{\mathbf{H}}^{\mathrm{PC}}=\sum_{\alpha=1}^{N} \mathbf{c}_{\alpha} \Psi_{\alpha}(\boldsymbol{\Xi})}_{N_{d} \text {-order gPCE }} \stackrel{\text { d. }}{\approx} \widetilde{\mathbf{H}},
$$

where $\stackrel{\text { d. }}{\approx}$ means the approximation in terms of distribution, $\mathbf{c}_{1}, \ldots, \mathbf{c}_{N}$ are vectors of coefficients in $\mathfrak{R}^{N_{g}}$ to be identified, and where $\Psi_{1}(\boldsymbol{\Xi}), \ldots, \Psi_{N}(\boldsymbol{\Xi})$ are the renumbered orthogonal Legendre polynomials, shifted to agree with the domain of random vector $\boldsymbol{\Xi},[0,1]^{N_{g}}$, and whose orders are lower than or equal to $N_{d}$. The number of polynomials of this expansion is $N=\left(N_{d}+N_{g}\right) ! / N_{g} ! N_{d} !$.

The coefficients $\mathbf{c}_{1}, \ldots, \mathbf{c}_{N}$ are identified in order to enhance the approximation in terms of distribution as stated by Eq. (33). During the identification process, the distribution of the random vector $\widetilde{\mathbf{H}}$ is estimated from its $m$ explicitly evaluated samples $\left\{\tilde{\boldsymbol{\eta}}^{(1)}, \ldots, \tilde{\boldsymbol{\eta}}^{(m)}\right\}$ using the multivariate kernel density estimation detailed in AppendixB. The identification of gPCE coefficients is detailed in AppendixC, and summarized in the following. There exists an isoprobabilistic transformation, the inverse Rosenblatt transformation [39], matching each sample of the random vector $\boldsymbol{\Xi}$ to a sample of the random vector $\widetilde{\mathbf{H}}$, and defined using cumulative distribution functions. The coefficients of the gPCE model are identified by projecting that inverse Rosenblatt transformation on the orthogonal Legendre polynomials [58]. That projection is numerically implemented by the Gauss quadrature rule with negligible computational cost.

Owing to the stochastic model, from the generated realizations of the distribution random vector $\boldsymbol{\Xi}$, the corresponding realizations of the random vector $\widetilde{\mathbf{H}}$ are obtained.

\subsubsection{Summary}

In this section, the methodology to construct the stochastic model of the random apparent adhesive contact forces, and its use to generate the realizations of these forces, are summarized.

The construction of the stochastic model for random apparent contact forces includes 3 steps,

(i) Acquiring input data, see the boxes indexed (II) and (III) in Fig. 8, which consists in

- Generating $m$ IID contacting surfaces with the size of the sub-contact domain $l_{1}^{\text {meso }} \times l_{2}^{\text {meso }}$ using the methodology developed in Section 2.2.1;

- Evaluating the $m$ corresponding apparent contact forces using the method described in Section 2.2 .2

- Parametrizing the evaluated apparent contact forces using the method described in Section 3.1 to obtain $m$ physical parameters vectors $\left\{\mathbf{v}^{(1)}, \ldots, \mathbf{v}^{(m)}\right\}$;

(ii) Processing input data, contributing to the task of the box (IV) in Fig. 8, by

- Evaluating the $m$ corresponding vectors $\left\{\mathbf{q}^{(1)}, \ldots, \mathbf{q}^{(m)}\right\}$ using Eq. (24); and

- Performing the linear dimension reduction which consists in evaluating the $m$ 4-dimensional vectors $\left\{\boldsymbol{\eta}^{(1)}, \ldots, \boldsymbol{\eta}^{(m)}\right\}$ using Eq. (29) and obtaining their reduced dimension $\left(N_{g} \leq 4\right)$ vectors $\left\{\widetilde{\boldsymbol{\eta}}^{(1)}, \ldots, \widetilde{\boldsymbol{\eta}}^{(m)}\right\}$, Eq. $(30)$;

(iii) Identifying the gPCE coefficients, see the box (IV) in Fig. 8, which consists in 
- For a chosen value of gPCE order $N_{d}$, defining the $\left(N_{d}+1\right)^{N_{g}}$ Gauss quadrature points on the hypercube $[0,1]^{N_{g}}$;

- For each Gauss point, evaluating the distribution of the random vector $\widetilde{\mathbf{H}}$ using the multivariate kernel density estimation method from the input data $\left\{\widetilde{\boldsymbol{\eta}}^{(1)}, \ldots, \widetilde{\boldsymbol{\eta}}^{(m)}\right\}$, see AppendixB, and then evaluating the inverse Rosenblatt transformation, see AppendixC;

- Evaluating the gPCE coefficients by projecting the inverse Rosenblatt transformation on the orthogonal Legendre polynomials system using the Gauss quadrature rule, see AppendixC.

Using the stochastic model, the apparent contact forces can be generated, see the box (V) in Fig. 8, by following 4 steps

(i) Generating realizations of the random vector $\boldsymbol{\Xi}$;

(ii) Evaluating the corresponding realizations of the random vector $\widetilde{\mathbf{H}}^{\mathrm{PC}}$ using Eq. (33);

(iii) Evaluating the corresponding realizations of the random vectors $\mathbf{Q}^{\mathrm{PC}}$ and $\mathbf{V}^{\mathrm{PC}}$ using Eq. (31) and Eq. (24) respectively;

(iv) Constructing the corresponding apparent contact forces using the analytical function described in Eq. (22).

The generated apparent contact forces are then integrated into the FE model at the structural scale to evaluate the realizations of the considered structural behaviors as discussed in Section 2.3, see the box (VI) in Fig. 8.

\section{Numerical verification on the stiction failure of micro cantilever beam}

The stochastic multiscale methods are applied to quantify the uncertainties of the humid stiction phenomenon of poly-silicon micro cantilever beams, as illustrated in Fig. 1. In this phenomenon, upon an initial contact, the menisci can develop, and the cantilever beams can permanently adhere to their substrate due to the capillary forces. The effect of $\mathrm{vdW}$ interaction is excluded in this section but is accounted for in Section 5. One of the important stiction configurations of micro cantilever beams is the S-shape configuration [59], see Fig. 1(a), in which the crack length defined as the horizontal distance from the crack tip to the clamp is an important quantity characterizing the considered problem. A shorter crack length indicates a higher adhesive energy. As it is discussed throughout the work, the phenomenon is uncertain, and the probabilistic behaviors of the quantities of interest, such as crack lengths and internal energies, need to be identified. Through the evaluation of the uncertainties of the quantities of interest, this section provides a numerical comparison of the two uncertainty propagation methods, the direct MCS method and the stochastic model-based method in terms of results and computational efficiency at Section 4.5.

\subsection{Problem setting}

A cantilever beam structure, illustrated in Fig. 1, is considered. The cantilever beams have a length $l=1500 \mu \mathrm{m}$, a width $w=30 \mu \mathrm{m}$, a thickness $t=2.62 \mu \mathrm{m}$, and are clamped at a height $h=1.9 \mu \mathrm{m}$ from their substrates. The considered cantilever beams and the substrate surfaces are both made of poly-silicon for which the Young's modulus $E=163 \mathrm{GPa}$, and water contact angles $\rho_{1}=\rho_{2} \simeq 0^{0}$. That configuration corresponds to the stiction experiments reported by DelRio et al. in [5]. In this section, humid condition with relative humidity $R H=85 \%$ is considered, and the $\mathrm{vdW}$ interaction is excluded.

Due to the lack of experimental data, the PSD function proposed by the authors in [14], which is a self-affine PSD function [60] of the equivalent surface constructed using the experimental data reported in 


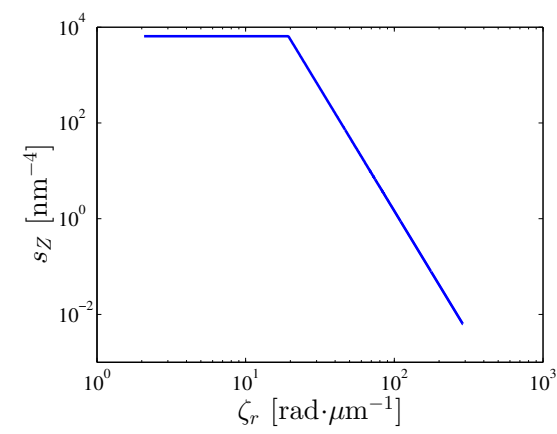

(a) For experiments reported in [5]

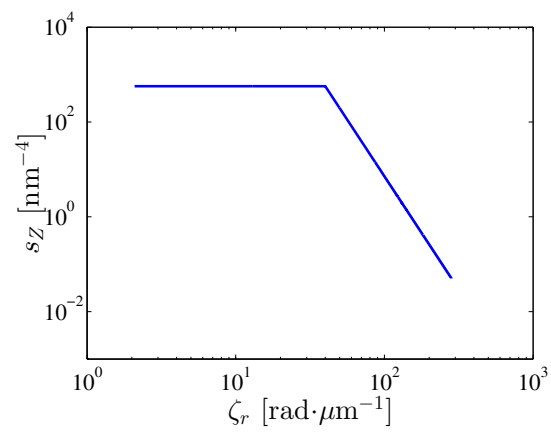

(b) For experiments reported in [9]

Figure 11: The PSD functions constructed using the data from the experiments reported in [5] and [9].

Table 2: The constructed PSD functions parameters and their generated surface properties corresponding to the experiments reported in [5] and [9]. The quantities with a "*" marker are the measurement data or parameter evaluations reported in [5, 9].

\begin{tabular}{l|c|c} 
Constructed PSD for tests reported by & DelRio et al. [5] & E. Soylemez et al. [9] \\
\hline \multicolumn{3}{c}{ Constructed PSD parameters } \\
\hline$\zeta_{\max }\left[\mathrm{rad} \cdot \mu \mathrm{m}^{-1}\right]$ & 289 & 283 \\
$\zeta_{r}^{0}\left[\mathrm{rad} \cdot \mu \mathrm{m}^{-1}\right]$ & 19.4 & 40 \\
$s_{Z}^{0}\left[\mathrm{~nm}^{4}\right]$ & $6.46 \times 10^{3}$ & $0.5 \times 10^{3}$ \\
$s_{Z}^{1}\left[\mathrm{~nm}^{4}\right]$ & $6.46 \times 10^{-3}$ & $50 \times 10^{-3}$ \\
\hline \multicolumn{3}{c}{ Generated surface statistical properties } \\
\hline Root mean square roughness [nm] & $3.5^{*}$ & $2.2{ }^{*}$ \\
1st order derivatives variance [-] & 0.0035 & 0.0057 \\
2nd order derivatives variance $\left[\mu \mathrm{m}^{-2}\right]$ & 9.1 & $45^{*}$ \\
\hline \multicolumn{2}{c}{ GW model $[15]$ parameters } \\
\hline Equivalent spherical asperity radius [nm] & 220 & $100{ }^{*}$ \\
Asperity height standard derivation [nm] & 3.3 & 2.1 \\
Asperity density $\left[\mu \mathrm{m}^{-2}\right]$ & 80.1 & 242
\end{tabular}

[5], is used. That PSD function is given by

$$
s_{Z}\left(\zeta_{r}\right)= \begin{cases}s_{Z}^{0} & \text { if } 0 \leq \zeta_{r}<\zeta_{r}^{0} ; \\ s_{Z}^{0}\left(\frac{\zeta_{r}}{\zeta_{r}^{0}}\right)^{\log _{10}\left(\frac{s_{Z}^{1}}{s_{z}^{0}}\right) / \log _{10}\left(\frac{\zeta_{\max }}{\zeta_{r}^{0}}\right)} & \text { if } \zeta_{r}^{0} \leq \zeta_{r} \leq \zeta_{\max } \\ 0 & \text { if } \zeta_{\max }<\zeta_{r} ;\end{cases}
$$

where $\zeta_{r}=\sqrt{\zeta_{1}^{2}+\zeta_{2}^{2}}$. The parameters of the PSD function constructed for the experiments reported in [5], and the properties of its generated surfaces, are reported in the 2nd column of Tab. 2. With that proposed PSD function, illustrated in Fig. 11(a), the contact surfaces can be generated using the methodology described in Section 2.2.1, a realization of which is illustrated in Fig. 3. The surfaces generated using the proposed PSD function are isotropic, stationary, and have the root mean square roughness of $\mathrm{rms}=3.5 \mathrm{~nm}$ and a correlation length of $l^{\mathrm{m}}=0.7[\mu \mathrm{m}]$ - the minimum length for which the autocorrelation function of the rough surface, defined in Eq. (6), is smaller than $1 \%$ of the variance of surface heights. Even though it is not applied in this paper, the parameters of the GW model [15] of the generated surfaces are also reported in Tab. 2 for informative purpose ${ }^{4}$. In Fig. 11 and Tab. 2, the PSD function constructed for the experiments

${ }^{4}$ In the GW model, a random surface with a Gaussian heigth distribution is modeled by a set of spherical asperities of 
reported in [9] is also reported for comparison purpose, as it will be used in Section 5 when the numerical results are validated with the experimental data reported in both publications [5, 9]. The quantities with a “*” marker reported in Tab. 2 are the measurement data and parameter evaluations reported in [5, 9], which are used as the matching conditions when constructing the PSD function. As the set of provided parameters is not enough to build the PSD, we complete it by considering reasonable values from other experimental surfaces processed using a similar fabrication methodology [4, 14].

In the following, the multiscale model of the adhesive cantilever beam is first analysed on a surface realization of the equivalent surface $\mathcal{S}$, before being coupled with the uncertainty quantification methods.

\subsection{Implementation of the multiscale FE model for micro cantilever beam undergoing stiction}

A surface $\mathcal{S}$ corresponding to the beam bottom surface of size $l \times w$ can be generated using the methodology described in Section 2.2.1 from the proposed PSD function in Eq. (34). To reduce the computational cost, for $R H=85 \%$, the length of the numerical beam is chosen as $l=600[\mu \mathrm{m}]$ (smaller than the experimental beams length of $1500[\mu \mathrm{m}])$, as the crack lengths obtained from that experiments at $R H=85 \%$ are within the 180-250 $[\mu \mathrm{m}]$ range. The surface is then divided into $N_{p}$ non-overlapping surfaces $\mathcal{S}^{r}$ of size $l^{\text {meso }} \times w$, with $r=1, \ldots, N_{p}$ and $N_{p}=l / l^{\text {meso }}$, corresponding to $N_{p}$ sub-contact domains. The value of $l^{\text {meso }}$ is chosen to be $3 \mu \mathrm{m}$ and the corresponding value of $N_{p}$ is 200 . That choice will be justified later in this section by a convergence analysis. The apparent adhesive contact forces are evaluated for each sub-contact domain by solving the meso-scale contact problem, see Fig. 2(b), using the methodology described in Section 2.2.2 and fitted via the analytical function defined by Eq. (22). They are then integrated into a 1D elastic FE model of cantilever beam using Eq. (17) to evaluate the corresponding structural behavior. In the FE model of cantilever beam, the stiffness matrix $[K]$, see Eq. (16), is calculated using Euler-Bernoulli beam theory. In the following, the main numerical features of the multiscale FE model of the micro cantilever beam are discussed.

Failure simulation process. The menisci develop when the micro cantilever beams touch their substrate due to external forces. Because the Laplace pressure inside the menisci pulls the beam, the contact zone propagates. This process can lead to the stiction of the cantilever beams to their substrate, see Fig. 1(a). In the present numerical model, to predict the stiction configuration of a micro cantilever beam with the shortest possible crack length, we perform a loading-unloading simulation. The process is illustrated in terms of the evaluation of the beam central line in Figs. 12(a,b) and in terms of the evaluation of beam internal energy in Fig. 12(c). The two phases of the process are described in the following.

(i) The cantilever beam is first loaded by an increasing external force applied at a distance, e.g. $60 \mu \mathrm{m}$, from the clamp. The external force pushes the cantilever beam into contact with the substrate, see Fig. 12(a). When a contact zone is initiated, the apparent adhesive contact forces are activated, pull the cantilever beam on its substrate, and, as a consequence, the contact zone develops. The loading process is ended when the loaded crack length reaches a certain value which is smaller than the expected minimum crack length. The pull-in phenomenon, see Figs. 12(a, c), characterized by the simultaneous increases of the internal energy resulting from the increase of the beam deformation, is observed during the loading process. Because the apparent contact forces of two different subcontact domains are different, the pull-in phenomenon occurs multiple times. That phenomenon is also experimental observed in [9].

(ii) The applied load is then gradually decreased to zero, see Fig. 12(b). At the end of the unloading process, the failure configuration can be obtained, see Fig. 12(d). As during the loading process, the pull-out phenomenon, see Fig. 12(b,c), characterized by the simultaneous decreases of the internal energy, can be observed multiple times during the unloading process.

identical radius. The parameters describing the spherical asperities -the equivalent radius, the variance of asperity heights, and the asperities density- are derived from the statistical properties of considered surfaces. 


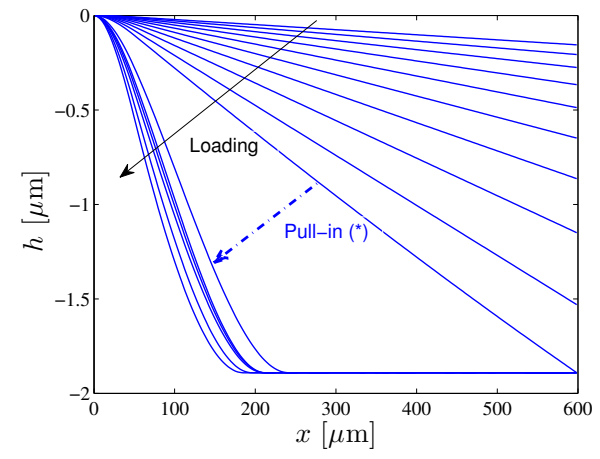

(a) Loading process

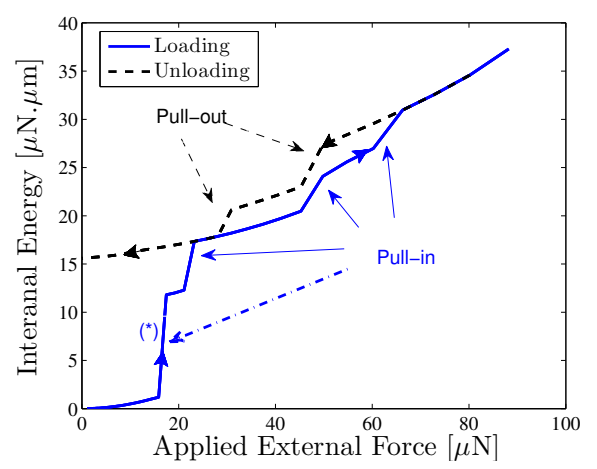

(c) Internal energy vs. applied load evolution

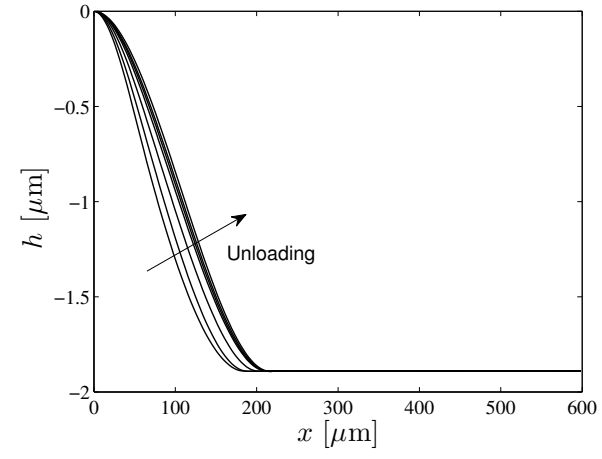

(b) Unloading process

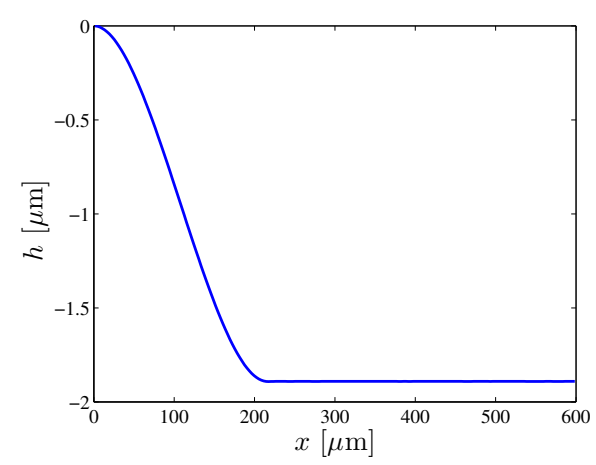

(d) Stiction configuration

Figure 12: The failure simulation process. (a) The evaluation of the beam central line during the loading process. The pull-in marked as $\left(^{*}\right)$ is illustrated in the evaluation of internal energy, see Fig. 12(c). (b) The evaluation of the beam central line during unloading process. (c) The evaluation of internal energy during loading and unloading process. The pull-in marked as (*) is illustrated in the evaluation of the beam central line, see Fig. 12(a). (d) The obtained configuration of beam central line at the stiction failure state.

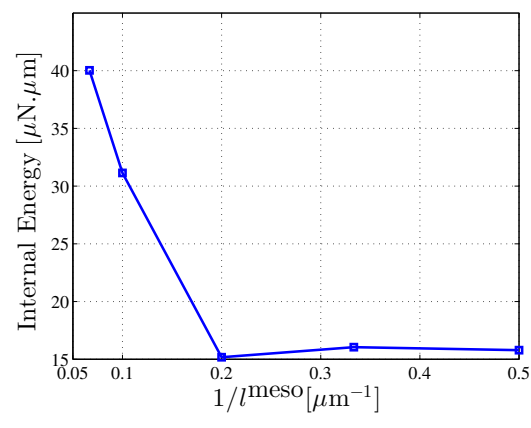

(a) Meso-scale length

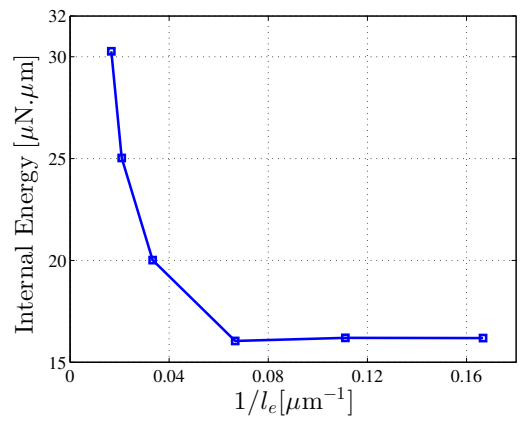

(b) Element length

Figure 13: The convergence analysis with respect of the meso-scale length $l^{\text {meso }}$ and of the element lengths $l_{e}$ in terms of the internal energies. (a) The convergence analysis with respect of the meso-scale length $l^{\text {meso }}$. (b) The convergence analysis with respect of the element lengths $l_{e}$ with meso-scale length $l^{\text {meso }}=3 \mu \mathrm{m}$.

Determining the meso-scale length. The meso-scale length $l^{\text {meso }}$, see Fig. 2(b), is determined to satisfy the two conditions (i) the variation of the beam central line displacement inside a sub-contact domain is negligible in comparison with the root mean square roughness, and (ii) $l^{\text {meso }} \geq l^{\mathrm{m}}$ in order for the homogenization 
process to hold. For the first condition, we can perform a convergence analysis of the beam internal energy in terms of $l^{\text {meso }}$. Fig. 13(a) illustrates the internal energy at the stiction configuration evaluated by the FE models using different meso-scale lengths $l^{\text {meso }}$ on the same realization of the contact surface $\mathcal{S}$. It is observed that the solution is converged when reducing the length $l^{\text {meso }}$ as the variation of the beam central line displacement inside a sub-contact domain is decreased. These numerical results suggest that we can choose the meso-scale length as $l^{\text {meso }}=3 \mu \mathrm{m}$ for which the condition $l^{\text {meso }}>l^{\mathrm{m}}=0.7 \mu \mathrm{m}$ is also verified.

Convergence of element length. The cantilever beam is descretized into finite elements of size $l_{e} \times w$. The convergence with respect of element size $l_{e}$ is illustrated in Fig. 13(b). These numerical results suggest that we can choose the element length as $l_{e}=15 \mu \mathrm{m}$. This means that for each element in the beam FE model, there are 5 integration points, corresponding to 5 apparent contact forces, to evaluate the equivalent nodal forces Eq. (17).

In the following, the uncertainty propagation methods, the direct MCS method and the stochastic modelbased method, described in Section 3 are coupled with the muti-scale FE model.

\subsection{Implementation of direct MCS multiscale method}

Using the random surface generator described in Section 2.2.1, one can generate $N_{M C}=1000$ surfaces $\mathcal{S}^{(k)}$ with $k=\left\{1, \ldots, N_{M C}\right\}$ of the beam size $l \times w$ from which the $N_{M C}$ corresponding structural behaviors are computed using the multiscale FE model as implemented in Section 4.2. The probabilistic behaviors of micro beams, e.g. distribution of crack lengths, are then evaluated and will be compared with the stochastic method in Section 4.5.

\subsection{Implementation of the stochastic model of random apparent adhesive contact forces}

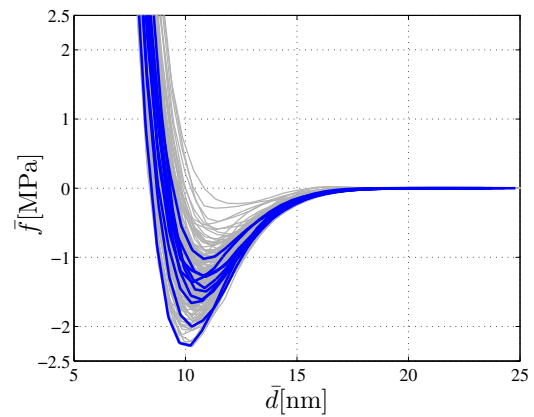

(a) 10 evaluated contact forces (highlighted ( among the other 200)

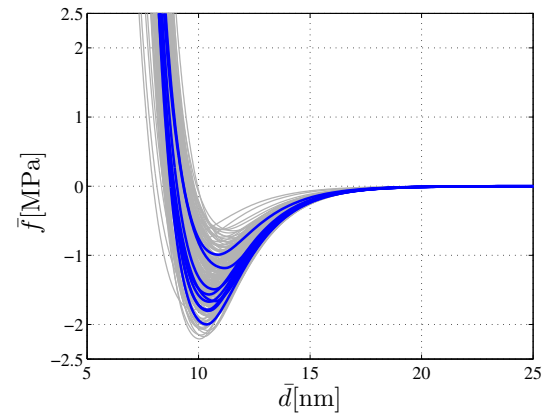

(b) 10 generated contact forces (highlighted among the other 200)

Figure 14: The IID realizations of the apparent adhesive contact forces explicitly evaluated by MCS (a), and generated by gPCE model (b).

The stochastic model of the random apparent adhesive contact forces is developed using an input data set of $m=5000\left(\ll N_{M C} \times N_{p}=1000 \times 200\right)$ explicitly evaluated apparent adhesive contact forces obtained by the MCS method. The evaluation of these contact forces is achieved by generating $m$ IID surfaces of size $l^{\text {meso }} \times w$ using the surface generator described in Section 2.2.1 from PSD function Eq. (34), and evaluating the $m$ corresponding apparent contact forces by the method developed in Section. 2.2.2. The choice of $m=5000$ is explained in Section 4.4.3. As discussed in Section 4.2, the meso-scale length $l^{\text {meso }}$ is determined by $3 \mu \mathrm{m}$.

Ten samples of the evaluated apparent contact forces are illustrated in Fig. 14(a). The equivalent distance at which the apparent contact force vanishes due to an equivalence between adhesive and repulsive forces ranges between $[8.3 \mathrm{~nm}-10 \mathrm{~nm}]$ corresponding to $[2.4 \times \mathrm{rms}-2.9 \times \mathrm{rms}]$. The physical contact area at the equivalent distance is observed to be $[0.2 \%-0.7 \%]$ of the apparent area and there are $[80-130]$ asperities 
entering into physical contact. Because the Laplace pressure $(22 \mathrm{MPa}$ for $R H=85 \%)$ is much smaller than the stiffness of the considered material polysilicon (163 GPa), the area occupied by menisci is much bigger than the physical contact area at the distance for which the force vanishes. That menisci area is observed to be in the range $[14 \%-29 \%]$ of the apparent area. Because of the small physical contact area and the small number of contacting asperities, there exist uncertainties on the apparent contact forces as it is observed in Fig. 14(a).

Using the parametrization process described in Section 3.1, the data set is represented by $m=5000$ samples $\left\{\mathbf{v}^{(1)}, \ldots, \mathbf{v}^{(m)}\right\}$ of the random parameters vector $\mathbf{V}$. In the following, the negligibility of the spatial correlation of the random contact forces is numerically validated, and then the stochastic model of the random vector $\mathbf{V}$ is constructed from those $m$ input samples and following the two previously detailed processes: input data processing and gPCE model.

\subsubsection{Spatial correlation of the apparent adhesive contact forces}

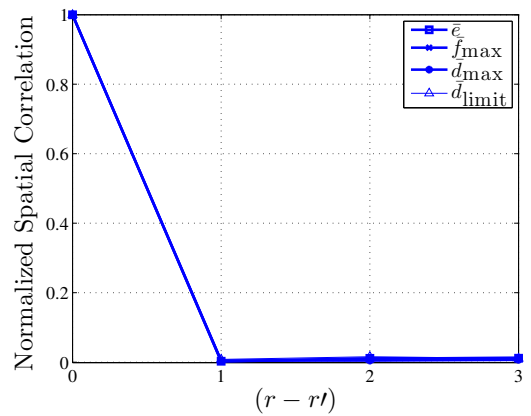

Figure 15: The normalized spatial correlations of the random variables $\bar{e}, \bar{f}_{\max }, \bar{d}_{\max }, \bar{d}_{\text {limit }}$ (The correlation curves are close to each other).

The normalized spatial correlations of components $V_{i}$ with $i=1, \ldots, 4$ of the random vector $\mathbf{V}$ are defined by

$$
\frac{\mathbb{E}\left[\left(V_{i}^{r}-\mathbb{E}\left(V_{i}\right)\right)\left(V_{i}^{r^{\prime}}-\mathbb{E}\left(V_{i}\right)\right)\right]}{\mathbb{E}\left[\left(V_{i}-\mathbb{E}\left(V_{i}\right)\right)^{2}\right]}, \quad \text { with }\left\{r, r^{\prime}\right\} \in\left\{1, \ldots, N_{p}\right\}^{2},
$$

and are illustrated in Fig. 15. This figure shows that the spatial correlations can be neglected, as in the multiscale model two adjacent sub-contact domains are such that $\left|r-r^{\prime}\right|=1$, see Fig. 7 .

\subsubsection{Identification of the reduced dimensions number}

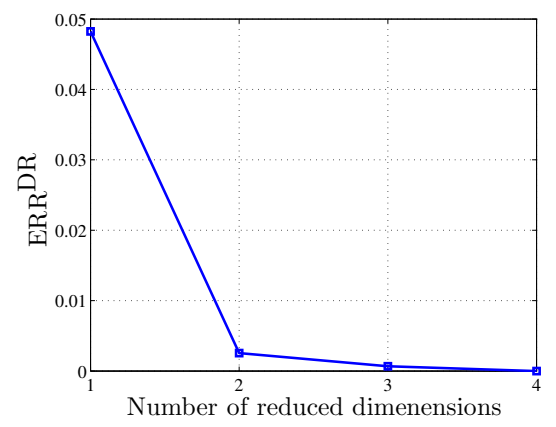

Figure 16: The error due to the dimension reduction process $\operatorname{Err}^{\mathrm{DR}}$.

To lower the effect of the curse of dimensionality, the linear dimension reduction reported in Section 3.2.1 is applied. From the input data set $\left\{\mathbf{v}^{(1)}, \ldots, \mathbf{v}^{(m)}\right\}$ with $m=5000$, the corresponding samples 
$\left\{\mathbf{q}^{(1)}, \ldots, \mathbf{q}^{(m)}\right\}$ of the random vector $\mathbf{Q}$ are evaluated by Eq. (24). Using the principal component transformation given by Eq. (29), the $m$ samples $\left\{\boldsymbol{\eta}^{(1)}, \ldots, \boldsymbol{\eta}^{(m)}\right\}$ of the orthogonal random vector $\mathbf{H}$ can be obtained. The dimension reduction error $\operatorname{Err}^{\mathrm{DR}}$ can then be evaluated using Eq. (32) and is illustrated in Fig. 16. These numerical results of the error Err ${ }^{\mathrm{DR}}$ suggest that the number of reduced dimensions can be chosen as $N_{g}=3$ with the corresponding error $\operatorname{Err}^{\mathrm{DR}}=0.07 \%$. Although there is only one dimension less, the number of the coefficients of gPCE model is lowered by $\left(N_{d}+4\right) / 4$ times, e.g $\left(N_{d}+4\right) / 4=4$ for $N_{d}=12$, which justifies the process. From $m$ samples $\left\{\boldsymbol{\eta}^{(1)}, \ldots, \boldsymbol{\eta}^{(m)}\right\}$, the corresponding reduced dimension vectors $\left\{\tilde{\boldsymbol{\eta}}^{(1)}, \ldots, \tilde{\boldsymbol{\eta}}^{(m)}\right\}$ are obtained as $\tilde{\boldsymbol{\eta}}^{(k)}=\left\{\eta_{1}^{(k)}, \eta_{2}^{(k)}, \eta_{3}^{(k)}\right\}$ with $k=1, \ldots, m$.

\subsubsection{Construction the gPCE representation}

The input data for the construction of the gPCE model are $m$ vectors $\tilde{\boldsymbol{\eta}}^{(k)}=\left\{\eta_{1}^{(k)}, \eta_{2}^{(k)}, \eta_{3}^{(k)}\right\}$ with $k=1, \ldots, m$. The value of $m$ is chosen such that the randomness of the vector $\widetilde{\boldsymbol{\eta}}$ is well captured with $m$ input vectors $\left\{\tilde{\boldsymbol{\eta}}^{(1)}, \ldots, \tilde{\boldsymbol{\eta}}^{(m)}\right\}$. During the coefficients identification of the gPCE model, the distribution of the random vector $\widetilde{\mathbf{H}}$ is approximated by the multivariate kernel density estimation method, see AppendixB. The larger the value of $m$, the better the approximation. In AppendixB.2, the convergence analysis shows that $m$ can be chosen by 5000 .

The identification of the gPCE model coefficients in Eq. (33) is detailed in AppendixC.2. The convergence analysis in AppendixC.2 shows that the truncated order of the gPCE model can be chosen as $N_{d}=12$. With that constructed gPCE model, the samples of the random vector $\widetilde{\mathbf{H}}^{\mathrm{PC}}$ are generated. The comparisons between random vector $\widetilde{\mathbf{H}}^{\mathrm{PC}}$, generated by gPCE model, and the reference one $\widetilde{\mathbf{H}}$, obtaining from $\left\{\tilde{\boldsymbol{\eta}}^{(1)}, \ldots, \tilde{\boldsymbol{\eta}}^{(m)}\right\}$, are illustrated in Fig. 17 in terms of the marginal and joint distributions. As it can be observed from the illustrations in Fig. 17 as well as through the errors analysis in AppendixC.2, the constructed 12-order gPCE model approximates well the distribution of the random vector $\mathbf{H}$.

\subsubsection{Identification of the probability of beam stiction failure using the constructed stochastic model}

With the direct MCS multiscale model implemented in Section 4.3, $N_{M C}=1000$ realizations of the beam failure structure are required in order to evaluate the distribution of the quantities of interest, e.g. the distribution of crack lengths, see the next subsection. Therefore, using the constructed stochastic model, we generate $N_{M C} \times N_{p}$ realizations of the reduced dimension random vector $\widetilde{\mathbf{H}}^{\mathrm{PC}}$ using Eq. (33), where $N_{M C}=1000$ is the number of FE simulations and $N_{p}=200$ is the number of sub-domains for the numerical beam length $l=600 \mu \mathrm{m}$. The corresponding realizations of the random vectors $\mathbf{Q}^{\mathrm{PC}}$ and of the random vector $\mathbf{V}^{\mathrm{PC}}$ are then successively evaluated using Eq. (31) and Eq. (27), respectively. The apparent adhesive contact forces are constructed from $\mathbf{V}^{\mathrm{PC}}$ using the analytical function described in Eq. (22). Ten generated apparent adhesive contact forces are illustrated in Fig. 14(b). Integrating each set of $N_{p}$ generated apparent contact forces into the FE model developed in Section. 4.2, the corresponding behavior, e.g. crack length, is evaluated. From the results set of $N_{M C}$ FE simulations, the statistical properties of the structural behavior are identified and will be compared with the direct MCS method in Section 4.5.

\subsection{Comparison between the two uncertainty propagation methods}

The two uncertainty propagation methods, the direct MCS method as the reference and the stochastic model-based method, see Fig. 8, are compared in terms of the distributions of the random apparent adhesive contact forces and of the distributions of the predicted quantities of interest. In addition, a comparison of the computational efficiency of the two methods is performed.

The distributions of random meso-scale apparent adhesive contact forces. As it is can be observed from Fig. 14, the explicitly calculated contact forces and the generated ones, and from Fig. 18, the comparison in terms of the distributions between the random parameters vector $\mathbf{V}^{\mathrm{PC}}$, simulated by the stochastic modelbased method, and the reference one $\mathbf{V}$ evaluated from the $m$ explicitly evaluated samples $\left\{\mathbf{v}^{(1)}, \ldots \mathbf{v}^{(m)}\right\}$, the stochastic model approximates accurately the distribution of the random vector $\mathbf{V}$. 


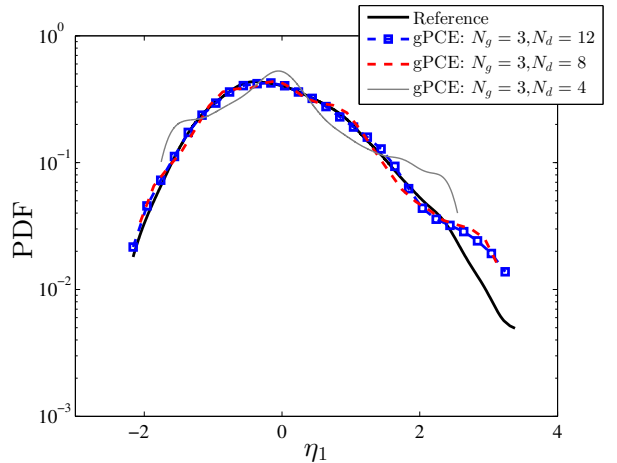

(a) PDF of $\eta_{1}$

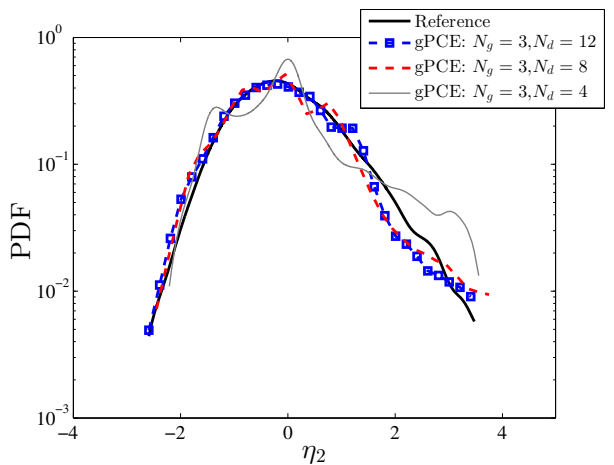

(c) PDF of $\eta_{2}$

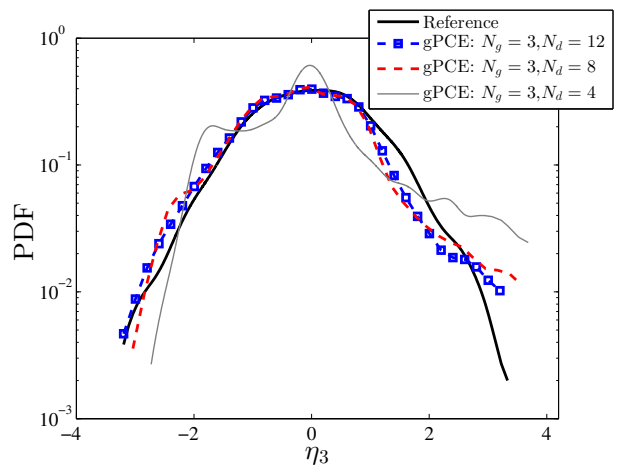

(e) PDF of $\eta_{3}$

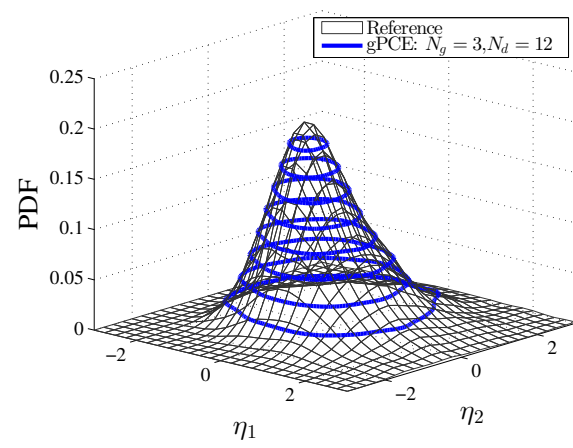

(b) Joint PDF of $\left\{\eta_{1}, \eta_{2}\right\}$

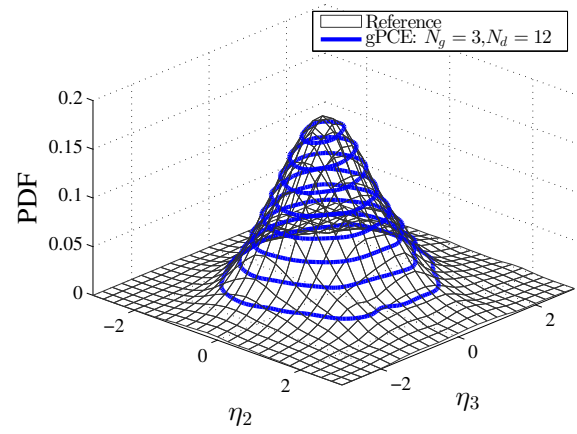

(d) Joint PDF of $\left\{\eta_{2}, \eta_{3}\right\}$

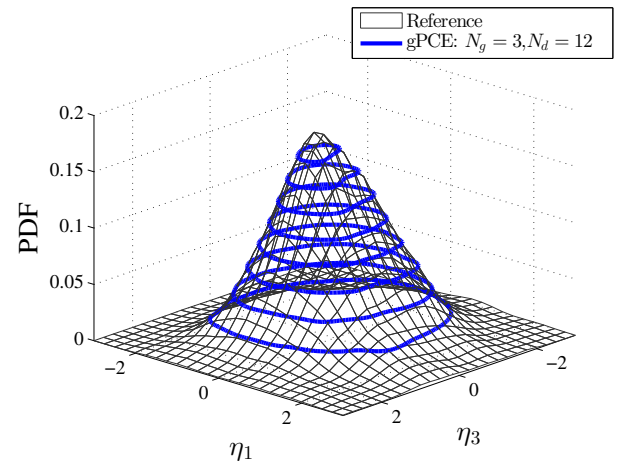

(f) Joint PDF of $\left\{\eta_{1}, \eta_{3}\right\}$

Figure 17: The convergence analysis of the distributions of the random vector $\widetilde{\mathbf{H}}^{\mathrm{PC}}$ in terms of gPCE model order $N_{d}$. (a,c,e) The comparisons between the marginal distributions, $\mathrm{p}_{H_{i}}$ with $i=1 . .3$, of $\widetilde{\mathbf{H}}^{\mathrm{PC}}$ obtained by the stochastic model with the reference ones obtained from the $m$ explicitly evaluated contact forces. (b,d,f) The comparisons between the joint bivariate distributions, $\mathrm{p}_{H_{i} H_{j}}$ with $\{i, j=1, \ldots, 3\}$, of $\widetilde{\mathbf{H}}^{\mathrm{PC}}$ obtained by the stochastic model with the reference ones obtained from the $m$ explicitly evaluated contact forces.

The distribution of crack lengths. By integrating the apparent adhesive contact forces, which can be either evaluated by the direct MCS method or generated using the constructed stochastic model, into a FE model of the cantilever beams we can evaluate the crack lengths, and quantify their uncertainty. The reported numerical crack length is defined as the length from the beam clamp to crack tip which is the separating point between the attached part on which the interaction forces are not zero and the unattached part with 


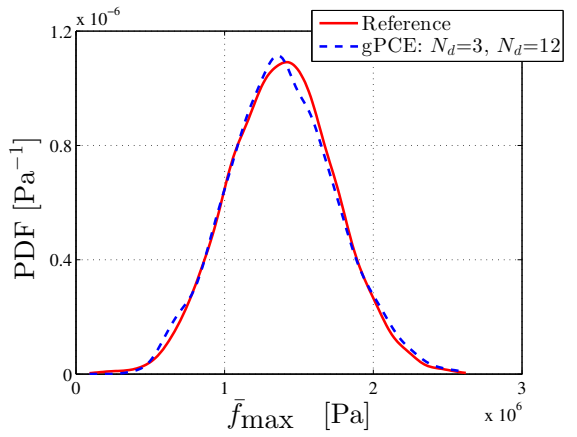

(a) PDF of $\bar{f}_{\max }$

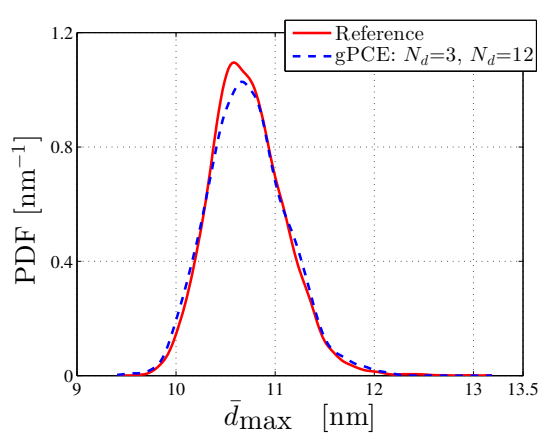

(c) PDF of $\bar{d}_{\max }$

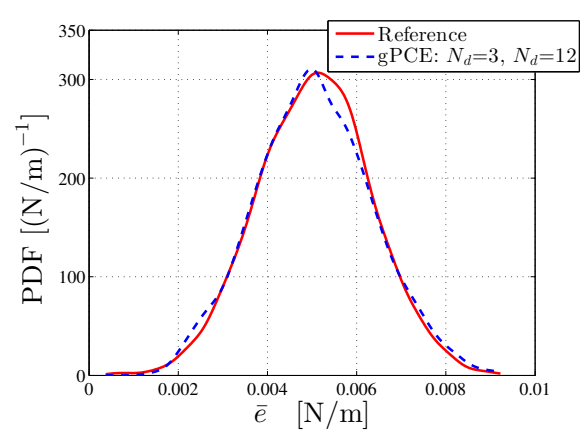

(b) PDF of $\bar{e}$

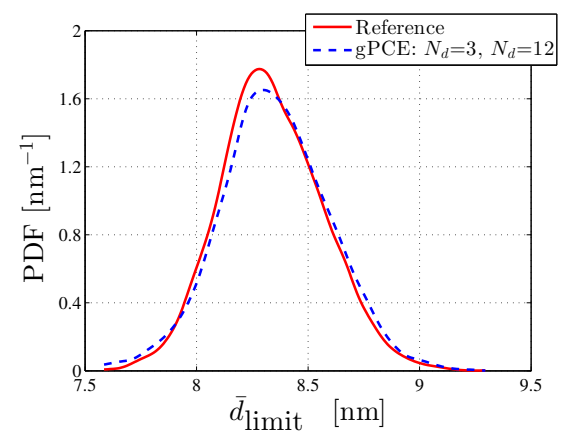

(d) PDF of $\bar{d}_{\text {limit }}$

Figure 18: The comparison between the marginal distributions of the random vector $\mathbf{V}^{\mathrm{PC}}$ generated by gPCE model and the references obtained from the $m$ explicitly evaluated contact forces.

vanishing interaction forces, see Fig. 1(a). The comparison between the results of the stochastic model and the reference ones in terms of statistical quantities and of the distribution of crack lengths are illustrated in Fig. 19. The numerical results illustrate that the stochastic model can predict the nominate properties of the crack length distribution.

Computational effectiveness. The stochastic model-based method is more computationally efficient than the direct MCS method. It is due to the facts that: (i) the number of contact forces needed to be evaluated to build the stochastic model, $m=5000$, is much smaller than the one required for direct MCS, e.g. $N_{M C} \times N_{p}=200000$ for $N_{M C}=1000$ beam samples and $N_{p}=200$ sub-contact domains; and, (ii) the coefficients of the gPCE model, Eq. (33), are efficiently evaluated using quadrature rules, see AppendixC. To identify the gPCE model coefficients from $m$ samples data, the computational time for one processor is 7 minutes. To evaluate the stiction failure configuration of one beam sample, a direct MCS method takes several hours $\left(\sim 16\right.$ hours) on one processor which mainly devotes for evaluating the $N_{p}=200$ apparent adhesive contact forces. In case of the stochastic model-based method, the constructed stochastic model is used to generate the apparent adhesive contact forces, therefore the computational time to obtain the stiction failure of one beam sample devotes only for running the FE model of the beam, and significantly reduces to 2-5 minutes in the same computing condition. For this case, by applying the developed stochastic model-based multiscale method, the overall computational time is reduced by $96 \%$ in comparison with direct MCS multiscale method.

\section{Comparison with experimental data}

The stochastic model-based multiscale method, implemented in Section 4, is applied to evaluate the stiction phenomenon of cantilever beams at different relative humidity levels. In order to quantify the 


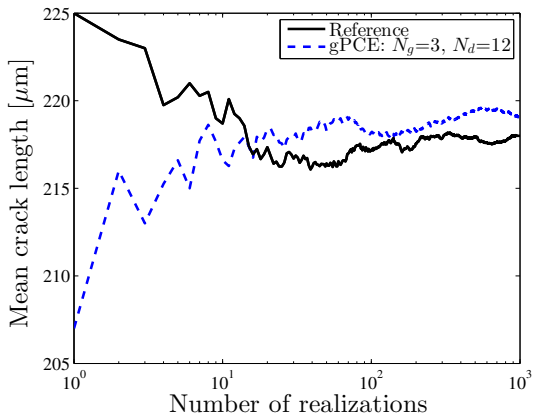

(a) Crack lengths mean

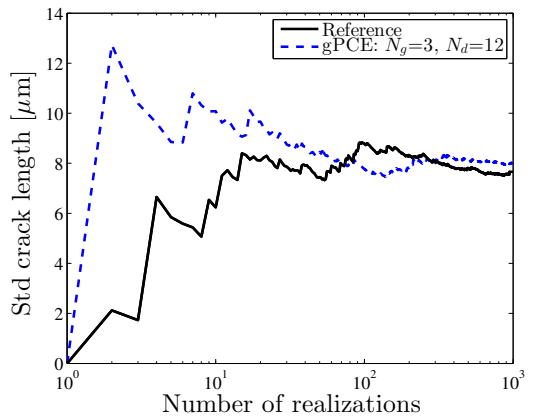

(b) Crack lengths STD

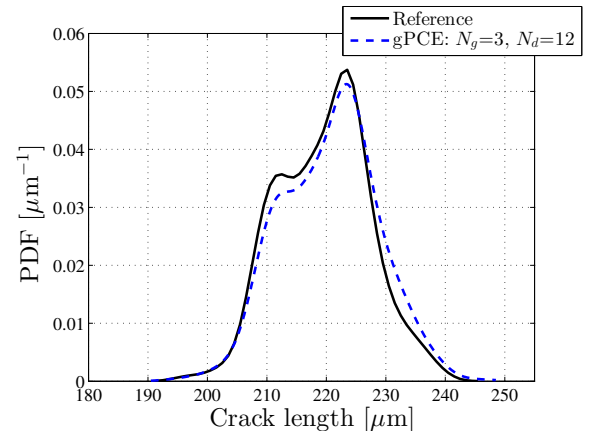

(c) PDF of crack lengths

Figure 19: The comparison between the two uncertainty propagation methods: stochastic model using gPCE and the direct MCS method as reference in terms of the distribution of crack lengths. (a) The convergence of the mean of crack length. (b) The convergence of the standard derivation (STD) of crack length. (c) The distribution of crack length.

Table 3: The comparison between the experimental data and the numerical results [5] in terms of average of crack length.

\begin{tabular}{l|ccccccccc} 
Relative humidity $R H[\%]$ & 0 & 30 & 45 & 55 & 65 & 70 & 85 & 90 & 95 \\
\hline \hline "Average" experimental crack lengths $[\mu \mathrm{m}]$ & 923 & 923 & 923 & 923 & 923 & 355 & 200 & 146 & 112 \\
\hline Average numerical crack lengths $[\mu \mathrm{m}]$ & 1266 & 1220 & 1151 & 983 & 749 & 620 & 219 & 150 & 111
\end{tabular}

stiction phenomenon de Boer et al. [59] have derived the apparent effective energy $\Gamma$ (in $\mathrm{J} / \mathrm{m}^{2}$ ) obtained from the crack lengths, $l_{s}$, as

$$
\Gamma=\frac{3}{2} E \frac{h^{2} t^{3}}{l_{s}^{4}}
$$

where $E$ is the Young's modulus. Using the developed stochastic model-based multiscale method, the crack lengths are evaluated and the corresponding apparent effective energies are obtained by Eq. (36).

Two experiments sets are considered, the first one developed by DelRio et al. in [5], and second one by E. Soylemez et al. in [9]. In both experimental measurements, the crack lengths of MEMS cantilever beams were measured for different humidity levels. One important input data of the developed method is the PSD function estimated from the measurements of the contacting surfaces. As we do not have access to

Table 4: The comparison between the experimental data and the numerical results [9] in terms of average of crack length.

\begin{tabular}{l|ccccccc} 
Relative humidity $R H[\%]$ & 0 & 35 & 55 & 65 & 70 & 80 & 90 \\
\hline \hline "Average" experimental crack lengths $[\mu \mathrm{m}]$ & 762 & 660 & 402 & 384 & 320 & 236 & 190 \\
\hline Average numerical crack lengths $[\mu \mathrm{m}]$ & 1293 & 1060 & 630 & 384 & 293 & 178 & 113
\end{tabular}




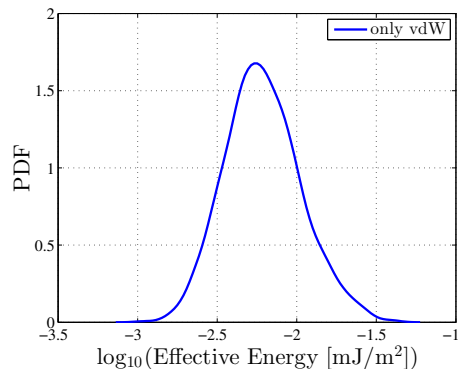

(a) $R H=0 \%$, only vdW interaction is accounted for.

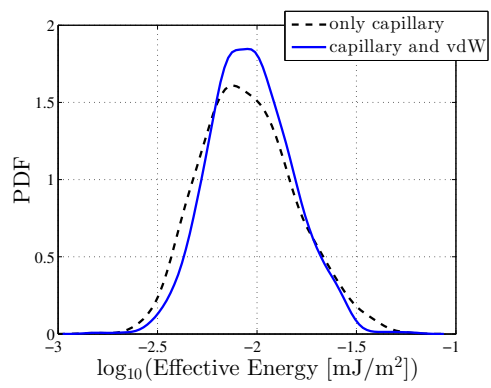

(c) $R H=45 \%$.

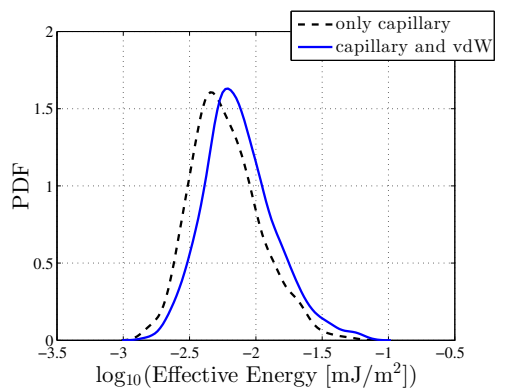

(b) $R H=30 \%$.

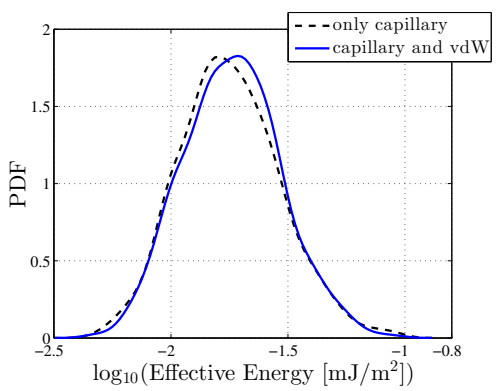

(d) $R H=55 \%$.

Figure 20: Comparison between the contributions of vdW forces and capillary forces to the distribution of apparent effective energies $\Gamma$ for the first set of experiments [9].

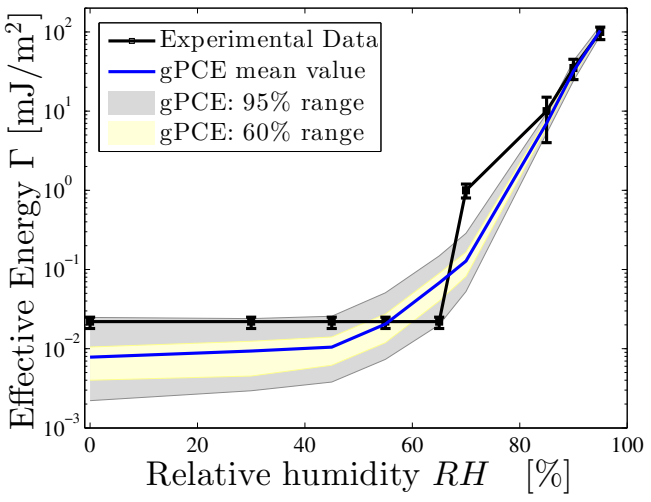

(a) Comparison with the experiments reported in [5]

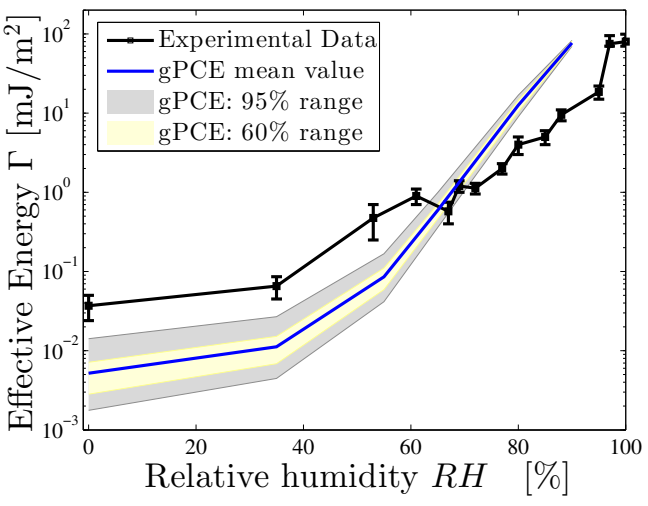

(b) Comparison with the experiments reported in [9]

Figure 21: The comparisons with the experimental data, reported in [5] (a) and in [9] (b), of the respective numerical results evaluated using the stochastic model-based multiscale method. 
the topology measurements, we construct the PSD functions from the information provided by the authors. The constructed PSD of the first set of experiments [5] has been already discussed in Section 4.1. For the second set of experiments, the assumption of self-affine surfaces is still applied, and the PSD is formulated as Eq. (34). However, the parameters are changed as reported in 3rd column of Tab. 2 to adapt with the information provided in paper [9], which is also reported in Tab. 2 by the "** marked quantities. The contructed PSD function of the second set of experiments is illustrated in Fig. 11(b).

In both cases, the numerical results take the vdW and capillary interactions into account for $R H<55 \%$, and neglect the vdW effect for higher humidity levels $R H \geq 55 \%$. As illustrated in Fig. 20, the capillary contribution to the adhesive behavior is dominant as compared to the vdW one for $R H \geq 55 \%$.

The comparisons are illustrated in Fig. 21 in terms of apparent effective energies $\Gamma$, and in Tabs. 3, 4 in terms of the means of crack lengths. We remark that the reported "average" experimental crack lengths are deduced from the average experimental apparent energies, extrapolated from their graphical illustration reported in [5, 9], using Eq. (36). Because the experiments were conducted using a small number of cantilever beams, while the numerical results are obtained from $N_{M C}=1000$ realizations, we draw the conclusion on the validation of stochastic model-based multiscale method with caution. Even though the PSD functions are constructed from limited information, the numerical results capture the main characteristics of the stiction phenomenon, i.e. the evolution of adhesive energies and their uncertainties in terms of humidity levels. The comparison is discussed in detail in the following.

(i) At high humidity levels, $R H \geq 55 \%$, the numerical results predict with accuracy the first set of experimental results [5], see Fig. 21(a) and Tab. 3. For the second set of experiments [9], the difference between the numerical and the experimental data at high humidity levels is not significant in comparison with the cantilever beam lengths $(2000-1050 \mu \mathrm{m})$, e.g. that difference is $58 \mu \mathrm{m}$ at $R H=80 \%$ corresponding to $2.9-5.5 \%$ of the cantilever beam lengths, see Tab. 4. Besides the problem of the indirect construction the PSD functions from incomplete information, these differences might also be explained by the fact that the reported experimental tests did not follow the similar loading-unloading process as in the numerical implementation of this work. Thus the pull-in/ pull-out interactions, see Fig. 12(a,b,c), cannot be observed completely during the test, and the experimental results do not necessarily conduct to the minimum crack lengths.

(ii) At low humidity levels, $R H<55 \%$, there exist discrepencies between the numerical predictions and the experimental data, see Fig. 21. It can result from the assumption of Gaussian distribution of the surface heights. As discussed in [13, 61], the Gaussian distribution assumption strongly affects the prediction of the numerical results, especially at low humidity levels when the ranges of vdW and capillary interactions are small in comparison with the surface roughness. Because of that assumption, the numerical results underestimated the adhesive energies [13,61], as it is also observed here in Fig. 21 and Tabs. 3, 4. Therefore, by improving the surface generator, the gap between numerical predictions and experimental data for low humidity levels might be reduced. In terms of implementation, the developed stochastic model-based multiscale method can directly adopt that improvement by considering other surface generators, e.g. a non-Gaussian one [62].

To improve the numerical results the following aspects should be accounted for.

(i) Estimate the PSD directly from AFM measurements of contacting surfaces.

(ii) Consider to replace the assumption of Gaussian distribution of surface heights, by a proper one corresponding to the measurements, i.e. applying Pearson distributions to account not only for the mean and the variance but also for the skewness and the kurtorsis.

(iii) Account for the other physical aspects, the absorbed surface layers [47], and other sources of uncertainties such as the distribution of contaminant particles on the surfaces [63, 64], when evaluating the apparent contact forces. 


\section{Conclusions}

For the considered physical problems of MEMS stiction, because the roughness of the contacting surfaces is comparable with the adhesive force ranges, and the length scale separation between the surface roughness and the apparent contact area is not fully satisfied, the computational multiscale method process should consider the uncertainties. In this work, we have developed a stochastic multiscale method that takes the roughness of the contacting surfaces into account in order to predict the probabilistic behaviors of micro structures involving adhesive contacts. The proposed method uses the random meso-scale apparent adhesive contact force as a scale bridge between the rough surfaces contact behaviors and the involved structural behaviors. The random apparent adhesive contact forces are integrated into a FE model of the studied structures to evaluate the structural behaviors in a probabilistic way.

To this end, the stochastic model-based multiscale methodology is proposed to avoid the explicitly evaluation of a huge number of apparent contact forces required by a direct MCS. In this method, the construction of the stochastic model (using gPCE) of the random apparent contact forces is the trading additional cost and is tailored in the present work. To construct the stochastic model, the random apparent contact force evolution (force vs. distance curve) is parametrized by the random vector of parameters on which a linear dimension reduction is performed to lessen the effect of the curse of dimensionality. A gPCE model is thus constructed to represent the reduced dimension random vector. The coefficients of the gPCE are identified efficiently using isoprobabilistic transformations constructed based on Rosenblatt transformation. Therefore, the proposed method is performed with an acceptable computational cost.

The methodology is validated through a comparison with the experimental data of the micro cantilever beam stiction. Even though the PSD functions are constructed indirectly from limited data, the numerical results well predict the trends of the adhesive energies and their uncertainty ranges. However, there are differences between numerical and experimental results at low humidity levels. These differences can be reduced by replacing the assumption of Gaussian distribution of the surface heights by the proper ones, e.g. Pearson distributions. In terms of implementation, the developed stochastic-model based multiscale method can fully adopt that improvement of surface heights distribution. Indeed, only the generator of the contacting surfaces needs to be changed. In addition, other physical effects such as the absorbed surface layer and other sources of uncertainties such as the distribution of contaminant particles on the surfaces should be investigated and accounted for.

\section{Acknowledgments}

The research has been funded by the Walloon Region under the agreement no 1117477 (CT-INT 201111-14) in the context of the ERA-NET MNT framework.

\section{AppendixA. The generator of rough surfaces using PSD function}

By assuming that the rough surface is a stationary and Gaussian random field, for a chosen value of the maximum wave number $\mu$, the normal height $Z^{(\mu)}(\theta)$ corresponding to the realization surface $\mathcal{S}(\theta)$ can be generated using the PSD function $s_{Z}$ following the works of Shinozuka [44] and Poiron and Soize [45], with

$$
\begin{aligned}
& z^{(\mu)}(\mathbf{x}, \theta)= \\
& \sqrt{2 \Delta \zeta^{2}} \mathfrak{R e}\left\{\sum_{l_{1}=1}^{\mu} \sum_{l_{2}=1}^{\mu} \beta_{\left(l_{1}, l_{2}\right)}(\theta) \sqrt{\frac{1}{(2 \pi)^{2}} s_{Z}\left(\zeta_{l_{1}}, \zeta_{l_{2}}\right)} \exp \left(\mathrm{i} x_{1} \zeta_{l_{1}}+\mathrm{i} x_{2} \zeta_{l_{2}}+\mathrm{i} \phi_{\left(l_{1}, l_{2}\right)}(\theta)\right)\right\}
\end{aligned}
$$

where

- the values $\left\{\left(\zeta_{l_{1}}, \zeta_{l_{2}}\right), \quad 1 \leq l_{1}, l_{2} \leq \mu\right\}$ are samplings of the wave number domain such that $\left\{\left(\zeta_{l_{1}}, \zeta_{l_{2}}\right)=\right.$ $\left.\left(-\zeta_{L}+\left(l_{1}-1\right) \Delta \zeta,-\zeta_{L}+\left(l_{2}-1\right) \Delta \zeta\right), 1 \leq l_{1}, l_{2} \leq \mu\right\}$, with $\Delta \zeta=2 \zeta_{L} / \mu$ 


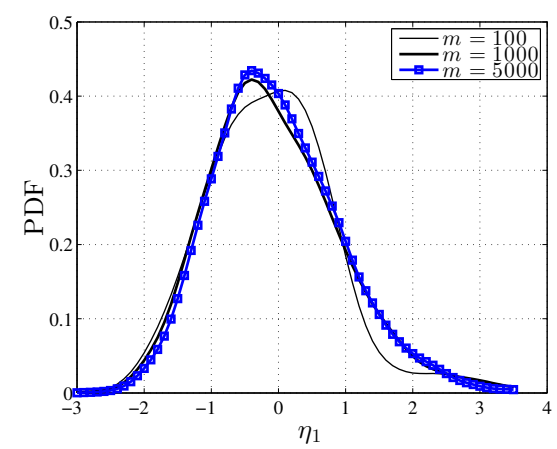

Figure B.22: The convergence with respect to $m$ when approximating the PDF of $\eta_{1}$ using the kernel density estimation.

- the values $\left\{\phi_{\left(l_{1}, l_{2}\right)}(\theta), \quad 1 \leq l_{1}, l_{2} \leq \mu\right\}$ are $\mu \times \mu$ independent realizations of a uniform random variable with values in $[0,2 \pi]$; and

- the values $\left\{\beta_{\left(l_{1}, l_{2}\right)}(\theta), \quad 1 \leq l_{1}, l_{2} \leq \mu\right\}$ are such that $\beta_{\left(l_{1}, l_{2}\right)(\theta)}=-\sqrt{-\log \left(\psi_{\left(l_{1}, l_{2}\right)}(\theta)\right)}$, where $\left\{\psi_{\left(l_{1}, l_{2}\right)}(\theta)\right.$, $1 \leq$ $\left.l_{1}, l_{2} \leq \mu\right\}$ are $\mu \times \mu$ independent realizations of a uniform random variable with values in $\left.] 0,1\right]$.

The implementations of Eqs. (9) and (A.1) are costly in terms of computational resources due to the double integration/ sum operations. To reduce the computational time, we can apply the fast Fourier transform (FFT) scheme developed for the rough surface cases as in [14].

\section{AppendixB. Multivariate kernel density estimation}

\section{AppendixB.1. Theory}

From the $m$ explicitly evaluated vectors $\left\{\mathbf{q}^{(1)}, \ldots, \mathbf{q}^{(m)}\right\}, m$ full dimension vector samples $\left\{\boldsymbol{\eta}^{(1)}, \ldots, \boldsymbol{\eta}^{(m)}\right\}$ and their corresponding reduced dimension vector samples $\left\{\tilde{\boldsymbol{\eta}}^{(1)}, \ldots, \tilde{\boldsymbol{\eta}}^{(m)}\right\}$ are evaluated using Eqs. (29, 30). Using $m$ samples $\left\{\tilde{\boldsymbol{\eta}}^{(1)}, \ldots, \tilde{\boldsymbol{\eta}}^{(m)}\right\}$, the probability density function $\mathrm{p}_{\widetilde{\mathbf{H}}}$ of the random vector $\widetilde{\mathbf{H}}$ can be approximated by $\hat{\mathrm{p}}_{\widetilde{\mathbf{H}}}$ evaluated using the multivariate kernel density estimation method [66] as

$$
\mathrm{p}_{\widetilde{\mathbf{H}}}(\tilde{\boldsymbol{\eta}}) \approx \hat{\mathrm{p}}_{\widetilde{\mathbf{H}}}(\tilde{\boldsymbol{\eta}})=\frac{1}{m} \sum_{k=1}^{m} \mathbb{K}_{[B]}\left(\tilde{\boldsymbol{\eta}}-\tilde{\boldsymbol{\eta}}^{(k)}\right),
$$

where $\mathbb{K}$ is the kernel function and $[B]$ is the bandwidth matrix. For a convenient choice, the kernel function can be chosen as the multivariate normal distribution function $\mathcal{N}(\mathbf{0},[B])$ with the covariance matrix $[B]$. In this case, one can use the thumb-up rule proposed by Scott $[66]$ for which the bandwidth matrix $[B]$ is a diagonal matrix given by

$$
[B]=m^{\frac{-2}{N_{g}+4}}\left[C_{\widetilde{\mathbf{H}}}\right]=m^{\frac{-2}{N_{g}+4}} I_{N_{g}},
$$

where $\left[C_{\widetilde{\mathbf{H}}}\right]=I_{N_{g}}$, and $N_{g}$ is the dimension of the vector $\tilde{\eta}$. With this choice of the kernel function, Eq. (B.1) is rewritten as

$$
\mathrm{p}_{\widetilde{\mathbf{H}}}(\tilde{\boldsymbol{\eta}}) \approx \hat{\mathrm{p}}_{\widetilde{\mathbf{H}}}(\tilde{\boldsymbol{\eta}})=\frac{1}{m} \sum_{k=1}^{m} \frac{1}{(2 \pi)^{N_{g} / 2} m^{-N_{g} /\left(N_{g}+4\right)}} \exp \left(-\frac{1}{2} \times \frac{\left\|\tilde{\boldsymbol{\eta}}-\tilde{\boldsymbol{\eta}}^{(k)}\right\|}{m^{-2 /\left(N_{g}+4\right)}}\right) .
$$

It can be proved that $\hat{\mathrm{p}}_{\widetilde{\mathbf{H}}}(\tilde{\boldsymbol{\eta}}) \stackrel{m \rightarrow+\infty}{\longrightarrow} \mathrm{p}_{\widetilde{\mathbf{H}}}[66]$. 
AppendixB.2. Convergence of problem stated in Section 4

The number $m$ of the explicitly evaluated samples, $\left\{\tilde{\boldsymbol{\eta}}^{(1)}, \ldots, \tilde{\boldsymbol{\eta}}^{(m)}\right\}$, is important when accomplishing the distribution approximation $\mathrm{p}_{\tilde{\mathbf{H}}} \approx \hat{\mathrm{p}}_{\tilde{\mathbf{H}}}$ stated in Eq. (B.3). As it is observed in Fig. B.22, the convergence is achieved when approximating the $1 \mathrm{D}$ probabilistic density function of $\eta_{1}$ with $m=5000$. Therefore, it is reasonable to chose $m=5000$.

\section{AppendixC. Construction of the stochastic model using gPCE representation}

\section{AppendixC.1. Theory}

A stochastic model is constructed in order to generate the realizations of the random vector $\widetilde{\mathbf{H}}$ using its $m$ explicitly evaluated samples $\left\{\tilde{\boldsymbol{\eta}}^{(1)}, \ldots, \tilde{\boldsymbol{\eta}}^{(m)}\right\}$, obtained using Eqs. $(29,30)$, as input data. The stochastic model is required to satisfy two conditions:

(i) The distribution of the generated realizations of the random vectors $\widetilde{\mathbf{H}}$ using the stochastic model is an approximation of the one estimated from the explicitly evaluated samples, Eq. (B.3); and

(ii) As the purpose of developing this stochastic model is to reduce the computational cost, the stochastic model must generate the random realizations with negligible computational cost, and the construction of that stochastic model must be computationally efficient.

To respect the first condition (i), in this paper the stochastic model is constructed based on the Rosenblatt transformation [39], notated here $\mathfrak{T}$, which is an isoprobabilistic transformation from the objective $N_{g^{-}}$ dimensional random vector $\widetilde{\mathbf{H}}$ to the $N_{g}$-dimensional random vector $\boldsymbol{\Xi}$ uniformly distributed on the unit hypercube $[0,1]^{N_{g}}, \boldsymbol{\Xi}=\mathfrak{T}(\widetilde{\mathbf{H}})$. To achieve the second condition (ii), because the Rosenblatt transformation, discussed in the following, requires a non-trivial computational effort to be evaluated, it is approximated by a gPCE model which contains only polynomial functions that can be evaluated with a negligible computational cost. In addition, the gPCE model is constructed in a computationally efficient way using the projection method [58].

Rosenblatt transformation. The Rosenblatt transformation [39] $\boldsymbol{\Xi}=\mathfrak{T}(\widetilde{\mathbf{H}})$ is detailed component by component by

$$
\begin{aligned}
\xi_{1} & =\mathbb{C}_{\widetilde{\mathbf{H}}}\left(\eta_{1}\right) \\
\xi_{2} & =\mathbb{C}_{\widetilde{\mathbf{H}}}\left(\eta_{2} \mid \eta_{1}\right) \\
& \ldots \\
\xi_{N_{g}} & =\mathbb{C}_{\widetilde{\mathbf{H}}}\left(\eta_{N_{g}} \mid \eta_{N_{g}-1}, \ldots, \eta_{1}\right),
\end{aligned}
$$

where $\left(\mathbb{C}_{\widetilde{\mathbf{H}}}\left(\eta_{i} \mid \eta_{i-1}, \ldots, \eta_{1}\right)\right) \mathbb{C}_{\widetilde{\mathbf{H}}}\left(\eta_{i}\right)$ are the (conditional) cumulative distribution functions of the random variable $H_{i}$ evaluated at $\eta_{i}$ and which are defined as

$$
\mathbb{C}_{\widetilde{\mathbf{H}}}\left(\eta_{i} \mid \eta_{i-1}, \ldots, \eta_{1}\right)=\int_{-\infty}^{\eta_{i}} \mathrm{p}_{\widetilde{\mathbf{H}}}\left(x \mid \eta_{i-1}, \ldots, \eta_{1}\right) \mathrm{d} x, \quad \text { with } i=1, \ldots, N_{g},
$$

with $\left(\mathrm{p}_{\widetilde{\mathbf{H}}}\left(\eta_{i} \mid \eta_{i-1}, \ldots, \eta_{1}\right)\right) \mathrm{p}_{\widetilde{\mathbf{H}}}\left(\eta_{i}\right)$ the (conditional) distribution functions of the random variable $H_{i}$ evaluated at $\eta_{i}$ and which are estimated using Eq. (B.3). Because of the strictly monotonic property of the cumulative distribution functions, the inverse of Rosenblatt transformation,

$$
\widetilde{\mathbf{H}}=\mathfrak{T}^{-1}(\boldsymbol{\Xi}),
$$

exists and is detailed component by component as

$$
\begin{aligned}
\eta_{1} & =\mathbb{C}_{\widetilde{\mathbf{H}}}^{-1}\left(\xi_{1}\right) \\
\eta_{2} & =\mathbb{C}_{\widetilde{\mathbf{H}}}^{-1}\left(\xi_{2} \mid \eta_{1}\right) \\
& \ldots \\
\eta_{N_{g}} & =\mathbb{C}_{\tilde{\mathbf{H}}}^{-1}\left(\xi_{N_{g}} \mid \eta_{N_{g}-1}, \ldots, \eta_{1}\right) .
\end{aligned}
$$


The inverse Rosenblatt transformation described in Eq. (C.3) fulfills the condition of approximating distribution between generated and explicitly evaluated samples of random vector $\widetilde{\mathbf{H}}$. With this transformation, one can generate samples of a uniform distribution random vector $\boldsymbol{\Xi}$, and the corresponding realizations of the random vector $\widetilde{\mathbf{H}}$ can be evaluated. However, to evaluate the inverse Rosenblatt transformation, it requires a non-trivial computational effort devoted to the evaluation of the cumulative distribution functions, Eq. (C.2). To achieve the goal of negligible computational cost when evaluating the stochastic model, the inverse Rosenblatt transformation is approximated using gPCE model formulated in terms of polynomial functions.

Approximation of the inverse Rosenblatt transformation using gPCE. The inverse Rosenblatt transformation in Eq. (C.3) is approximated using a truncated gPCE [34, 35, 67] as

$$
\underbrace{\widetilde{\mathbf{H}}=\mathfrak{T}^{-1}(\boldsymbol{\Xi})}_{\text {Rosenblatt transformation }} \approx \underbrace{\widetilde{\mathbf{H}}^{\mathrm{PC}}=\sum_{\alpha=1}^{N} \mathbf{c}_{\alpha} \Psi_{\alpha}(\boldsymbol{\Xi})}_{N_{d} \text {-order gPCE }},
$$

where $\mathbf{c}_{1}, \ldots, \mathbf{c}_{N}$ are vectors of coefficients in $\mathfrak{R}^{N_{g}}$, and where $\Psi_{1}(\boldsymbol{\Xi}), \ldots, \Psi_{N}(\boldsymbol{\Xi})$ are the renumbered orthogonal Legendre polynomials, shifted to agree with the domain of random vector $\boldsymbol{\Xi},[0,1]^{N_{g}}$, and whose orders are less than or equal to $N_{d}$ with $N_{d}$ the order of the truncated gPCE. The number of polynomials of this expansion is $N=\left(N_{d}+N_{g}\right) ! / N_{g} ! N_{d} !$. The chaos polynomials $\Psi_{\alpha}(\boldsymbol{\Xi})$ are $\mathrm{p}_{\Xi}$-orthonormal, i.e.,

$$
\int_{[0,1]^{N g}} \Psi_{\alpha}(\boldsymbol{\xi}) \Psi_{\beta}(\boldsymbol{\xi}) \mathrm{p}_{\boldsymbol{\Xi}}(\boldsymbol{\xi}) \mathbf{d} \boldsymbol{\xi}=\delta_{\alpha \beta},
$$

where $\mathrm{p}_{\boldsymbol{\Xi}}=1$ is the probabilistic density function of random vector $\boldsymbol{\Xi}$, and $\delta_{\alpha \beta}$ is the Kronecker symbol. Thanks to the $\mathrm{p}_{\Xi^{-}}$-orthonormal property of the polynomial chaos system stated in Eq. (C.6), the coefficients $\mathbf{c}_{\alpha}$ can be evaluated by projecting Eq. (C.5) on the polynomial chaos system [58]

$$
\mathbf{c}_{\alpha}=\int_{[0,1]^{N g}} \mathfrak{T}^{-1}(\boldsymbol{\xi}) \Psi_{\alpha}(\boldsymbol{\xi}) \mathrm{d} \boldsymbol{\xi} .
$$

Remarks on the implementation: In terms of numerical implementation, the integration in Eq. (C.7) is efficiently evaluated using quadrature rules [68], e.g. using the Gauss quadrature rule with $\left(N_{d}+1\right)^{N_{g}}$ integral points as

$$
\mathbf{c}_{\alpha} \approx \sum_{i=1}^{\left(N_{d}+1\right)^{N_{g}}} \widehat{\mathfrak{T}}^{-1}\left(\boldsymbol{\xi}_{i}^{\mathrm{G}}\right) \Psi_{\alpha}\left(\boldsymbol{\xi}_{i}^{\mathrm{G}}\right) w_{i}, \quad \text { with } \quad \sum_{i=1}^{\left(N_{d}+1\right)^{N_{g}}} w_{i}=1,
$$

where $\widehat{\mathfrak{T}}^{-1}$ is the approximation of inverse Rosenblatt transformation $\mathfrak{T}^{-1}$ for which the probability density function and cumulative distribution functions are evaluated using multivariate kernel density estimation, Eq. (B.1), and $\left\{\boldsymbol{\xi}_{1}^{\mathrm{G}}, \ldots, \boldsymbol{\xi}_{\left(N_{d}+1\right)^{N_{g}}}^{\mathrm{G}}\right\}$ are the $\left(N_{d}+1\right)^{N_{g}}$ integral points defined on the hypercube $[0,1]^{N_{g}}$ and associated with the weights $\left\{w_{1}, \ldots, w_{\left(N_{d}+1\right)^{N_{g}}}\right\}$. In addition, the linear interpolation technique can be used to perform the inverse operation in Eq. (C.8) [58].

Error estimation. In order to quantify the convergence of the gPCE model in terms of the order of the polynomials chaos representation $N_{d}$, the mean integral square errors are considered,

$$
\begin{gathered}
\operatorname{relMISE}_{i}^{\mathrm{PC}}\left(N_{d}\right)=\frac{\int_{[0,1]^{N_{g}}}\left(\widehat{\mathfrak{T}}_{i}^{-1}(\boldsymbol{\xi})-\sum_{\alpha=1}^{N} c_{\alpha i} \Psi_{\alpha}(\boldsymbol{\xi})\right)^{2} \mathrm{~d} \boldsymbol{\xi}}{\int_{[0,1]}\left(\widehat{\mathfrak{T}}_{i}^{-1}(\boldsymbol{\xi})\right)^{2} \mathrm{~d} \boldsymbol{\xi}}, \quad \text { with } \quad i=1 . . N_{g} ; \\
\operatorname{relMISE}^{\mathrm{PC}}\left(N_{d}\right)=\frac{\int_{[0,1]^{N_{g}}}\left\|\widehat{\mathfrak{T}}^{-1}(\boldsymbol{\xi})-\sum_{\alpha=1}^{N} \mathbf{c}_{\alpha} \Psi_{\alpha}(\boldsymbol{\xi})\right\|^{2} \mathrm{~d} \boldsymbol{\xi}}{\int_{[0,1]^{N_{g}}}\left\|\widehat{\mathfrak{T}}^{-1}(\boldsymbol{\xi})\right\|^{2} \mathrm{~d} \boldsymbol{\xi}}
\end{gathered}
$$

These errors relMISE ${ }_{i}^{\mathrm{PC}}$ and relMISE ${ }^{\mathrm{PC}}$ are also evaluated using the Gauss quadrature rule. 


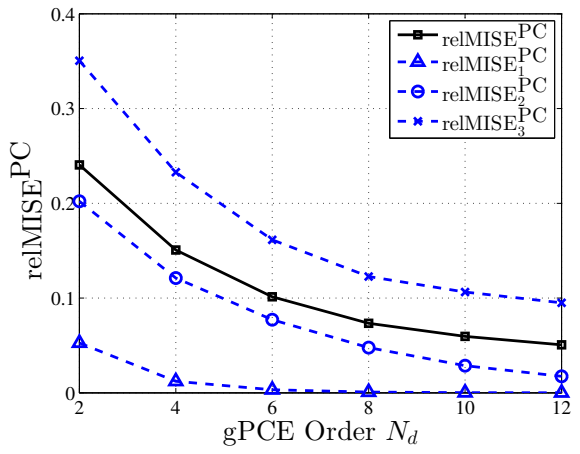

(a) Mean square integral errors

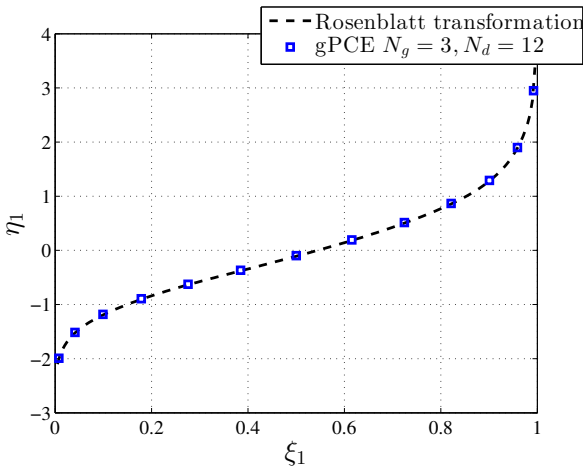

(b) $\mathfrak{T}_{1}^{-1}, \operatorname{relMISE}_{1}\left(N_{d}=12\right)=0.003 \%$

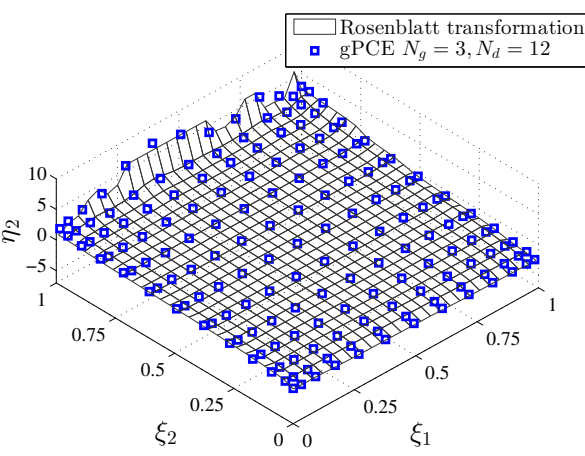

(c) $\mathfrak{T}_{2}^{-1}, \operatorname{relMISE}_{2}\left(N_{d}=12\right)=1.7 \%$

Figure C.23: The convergence analysis of gPCE model order $N_{d}$. (a) The mean square integral error defined in Eqs. (C.9-C.10). (b-c) The comparisons between the Rosenblatt transformation and the gPCE model.

AppendixC.2. Coefficients identification and convergence analysis of gPCE model stated in Section 4

With the input of $m=5000$ reduced dimension $\left(N_{g}=3\right)$ vectors $\left\{\tilde{\boldsymbol{\eta}}^{(1)}, \ldots, \tilde{\boldsymbol{\eta}}^{(m)}\right\}$, one can access to the approximations of the probability density function, (conditional) cumulative functions, and (inverse) Rosenblatt transformation using the multivariate kernel density estimation method, see AppendixB. The choice of $m=5000$ results from the convergence analysis reported in AppendixB.2. To identify the coefficients of the gPCE model, see Eq (33) for the compact form and Eq (C.5) for the detailed form, the inverse Rosenblatt transformation is projected on the polynomial chaos system, Eq. (C.7). The integrations in Eq. (C.7) are efficiently achieved using Gauss quadrature rule with $\left(N_{d}+1\right)^{N_{g}}$ integral points, Eq. (C.8) .

To study the convergence of the gPCE model in terms of the truncated order of gPCE model $N_{d}$, one can consider two aspects: (i) the mean square integral errors defined in Eqs. (C.9-C.10), see Fig. C.23(a); (ii) the comparison of gPCE model and Rosenblatt transformation, Eq. (C.5), see Figs. C.23(b,c) for $\left\{\mathfrak{T}_{1}^{-1}, \mathfrak{T}_{2}^{-1}\right\}$. As it can be observed from Figs. C.23, the constructed 12-order gPCE model can approximate the randomness of the reduced dimension random vector $\widetilde{\mathbf{H}}$ with $\operatorname{relMISE}^{\mathrm{PC}}\left(N_{d}=12\right)=5 \%$.

[1] W. M. van Spengen, Mems reliability from a failure mechanisms perspective, Microelectronics Reliability 43 (7) (2003) 1049-1060.

[2] W. M. Van Spengen, R. Puers, I. De Wolf, On the physics of stiction and its impact on the reliability of microstructures, Journal of Adhesion Science and Technology 17 (4) (2003) 563-582.

[3] W. M. Van Spengen, R. Puers, I. De Wolf, A physical model to predict stiction in mems, Journal of Micromechanics and Microengineering 12 (5) (2002) 702.

[4] M. de Boer, Capillary adhesion between elastically hard rough surfaces, Experimental Mechanics 47 (2007) $171-183$. doi:10.1007/s11340-006-0631-z.

URL http://dx.doi.org/10.1007/s11340-006-0631-z

[5] F. W. DelRio, M. L. Dunn, M. P. de Boer, Van der waals and capillary adhesion of polycrystalline silicon micromachined surfaces, in: B. Bhushan (Ed.), Scanning Probe Microscopy in Nanoscience and Nanotechnology 3, NanoScience and 
Technology, Springer Berlin Heidelberg, 2013, pp. 363-393. doi:10.1007/978-3-642-25414-7-14.

URL http://dx.doi.org/10.1007/978-3-642-25414-7-14

[6] L. Wu, V. Rochus, L. Noels, J. C. Golinval, Influence of adhesive rough surface contact on microswitches, Journal of Applied Physics 106 (11) (2009) 113502. doi:10.1063/1.3260248.

URL http://link.aip.org/link/?JAP/106/113502/1

[7] L. Wu, L. Noels, V. Rochus, M. Pustan, J.-C. Golinval, A micro - macro approach to predict stiction due to surface contact in micro electro-mechanical systems, Journal of Microelectromechanical Systems 20 (4) (2011) 976 -990. doi:10.1109/JMEMS.2011.2153823.

[8] L. Wu, J.-C. Golinval, L. Noels, A micro-model for elasto-plastic adhesive contact in micro-switches: Application to cyclic loading, Tribology International 57 (0) (2013) 137 - 146. doi:http://dx.doi.org/10.1016/j.triboint.2012.08.003. URL http://www.sciencedirect.com/science/article/pii/S0301679X12002757

[9] E. Soylemez, M. P. de Boer, Crack healing between rough polycrystalline silicon hydrophilic surfaces in n-pentanol and water vapors, Tribology Letters 59 (1) (2015) 1-12.

[10] M. de Boer, P. de Boer, Thermodynamics of capillary adhesion between rough surfaces, Journal of Colloid and Interface Science 311 (1) (2007) 171 - 185. doi:10.1016/j.jcis.2007.02.051. URL http://www.sciencedirect.com/science/article/pii/S0021979707002408

[11] W. M. van Spengen, A physical model to describe the distribution of adhesion strength in mems, or why one mems device sticks and another identical one does not, Journal of Micromechanics and Microengineering 25 (12) (2015) 125012 URL http://stacks.iop.org/0960-1317/25/i=12/a=125012

[12] D. Bachmann, S. Kühne, C. Hierold, Determination of the adhesion energy of mems structures by applying weibull-type distribution function, Sensors and Actuators A: Physical 132 (1) (2006) 407-414.

[13] E. Soylemez, M. P. de Boer, van der waals force-induced crack healing in dry rough interfaces, Journal of Physics D: Applied Physics 49 (7) (2016) 075303.

[14] T.-V. Hoang, L. Wu, S. Paquay, A. Obreja, R. Voicu, R. Müller, J.-C. Golinval, L. Noels, A probabilistic model for predicting the uncertainties of the humid stiction phenomenon on hard materials, Journal of Computational and Applied Mathematics 289 (2015) 173-195.

[15] J. A. Greenwood, J. B. P. Williamson, Contact of nominally flat surfaces, Proceedings of the Royal Society of London. Series A. Mathematical and Physical Sciences 295 (1442) (1966) 300-319. doi:10.1098/rspa.1966.0242.

[16] J. Greenwood, J. Wu, Surface roughness and contact: An apology, Meccanica 36 (6) (2001) 617-630. doi:10.1023/A:1016340601964. URL http://dx.doi.org/10.1023/A1016340601964

[17] R. Ardito, A. Corigliano, A. Frangi, F. Rizzini, Advanced models for the calculation of capillary attraction in axisymmetric configurations, European Journal of Mechanics - A/Solids 47 (0) (2014) 298 - 308. doi:http://dx.doi.org/10.1016/j.euromechsol.2014.05.002.

URL http://www.sciencedirect.com/science/article/pii/S0997753814000679

[18] R. Ardito, A. Corigliano, A. Frangi, Modelling of spontaneous adhesion phenomena in microelectro-mechanical systems, European Journal of Mechanics - A/Solids $39 \quad(0) \quad(2013) \quad 144 \quad-\quad 152$. doi:http://dx.doi.org/10.1016/j.euromechsol.2012.11.008.

URL http://www.sciencedirect.com/science/article/pii/S0997753812001489

[19] S. Hyun, M. O. Robbins, Elastic contact between rough surfaces: Effect of roughness at large and small wavelengths, Tribology International 40 (10) (2007) 1413-1422.

[20] L. Pei, S. Hyun, J. Molinari, M. O. Robbins, Finite element modeling of elasto-plastic contact between rough surfaces, Journal of the Mechanics and Physics of Solids 53 (11) (2005) 2385 - 2409. doi:http://dx.doi.org/10.1016/j.jmps.2005.06.008. URL http://www.sciencedirect.com/science/article/pii/S0022509605001225

[21] B. Luan, M. O. Robbins, Contact of single asperities with varying adhesion: comparing continuum mechanics to atomistic simulations, Physical Review E 74 (2) (2006) 026111.

[22] W. Sun, The dynamic effect on mechanical contacts between nanoparticles, Nanoscale 5 (2013) $12658-12669$. doi:10.1039/C3NR04354A. URL http://dx.doi.org/10.1039/C3NR04354A

[23] W. Sun, Y. Li, W. Xu, Y.-W. Mai, Interactions between crystalline nanospheres: comparisons between molecular dynamics simulations and continuum models, RSC Advances 4 (2014) 34500-34509. doi:10.1039/C4RA05480F. URL http://dx.doi.org/10.1039/C4RA05480F

[24] B. Luan, M. O. Robbins, Hybrid atomistic/continuum study of contact and friction between rough solids, Tribology letters 36 (1) (2009) 1-16.

[25] P. R. Nayak, Random process model of rough surfaces, Journal of Tribology 93 (3) (1971) 398-407. doi:10.1115/1.3451608.

[26] H. Hertz, Ueber die berührung fester elastischer körper., Journal für die reine und angewandte Mathematik 92 (1882) 156-171. URL http://eudml.org/doc/148490

[27] K. L. Johnson, K. Kendall, A. D. Roberts, Surface energy and the contact of elastic solids, Proceedings of the Royal Society of London. A. Mathematical and Physical Sciences 324 (1558) (1971) 301-313. doi:10.1098/rspa.1971.0141. URL http://rspa.royalsocietypublishing.org/content/324/1558/301.abstract

[28] B. Derjaguin, V. Muller, Y. Toporov, Effect of contact deformations on the adhesion of particles, Journal of Colloid and Interface Science 53 (2) (1975) 314 - 326. doi:10.1016/0021-9797(75)90018-1.

URL http://www.sciencedirect.com/science/article/pii/0021979775900181

[29] D. Maugis, Adhesion of spheres: The jkr-dmt transition using a dugdale model, Journal of Colloid and Interface Science 
150 (1) (1992) 243 - 269. doi:10.1016/0021-9797(92)90285-T.

URL http://www.sciencedirect.com/science/article/pii/002197979290285T

[30] C. Robert, G. Casella, Monte Carlo statistical methods, Springer Science \& Business Media, 2013.

[31] A. Clément, C. Soize, J. Yvonnet, Computational nonlinear stochastic homogenization using a nonconcurrent multiscale approach for hyperelastic heterogeneous microstructures analysis, International Journal for Numerical Methods in Engineering 91 (8) (2012) 799-824.

[32] A. Clément, C. Soize, J. Yvonnet, Uncertainty quantification in computational stochastic multiscale analysis of nonlinear elastic materials, Computer Methods in Applied Mechanics and Engineering 254 (2013) 61-82.

33] V. Lucas, J.-C. Golinval, S. Paquay, V.-D. Nguyen, L. Noels, L. Wu, A stochastic computational multiscale approach; application to mems resonators, Computer Methods in Applied Mechanics and Engineering 294 (2015) 141-167.

[34] R. G. Ghanem, P. D. Spanos, Stochastic finite elements: a spectral approach, Courier Corporation, 2003.

[35] C. Soize, R. Ghanem, Physical systems with random uncertainties: chaos representations with arbitrary probability measure, SIAM Journal on Scientific Computing 26 (2) (2004) 395-410.

[36] M. Arnst, R. Ghanem, C. Soize, Identification of bayesian posteriors for coefficients of chaos expansions, Journal of Computational Physics 229 (9) (2010) 3134-3154.

[37] C. Soize, Stochastic models of uncertainties in computational mechanics, American Society of Civil Engineers Reston, 2012.

[38] C. Desceliers, R. Ghanem, C. Soize, Maximum likelihood estimation of stochastic chaos representations from experimental data, International Journal for Numerical Methods in Engineering 66 (6) (2006) 978-1001.

[39] M. Rosenblatt, Remarks on a multivariate transformation, The annals of mathematical statistics (1952) 470-472.

[40] K. L. Johnson, K. L. Johnson, Contact mechanics, Cambridge university press, 1987.

[41] İ. Temizer, P. Wriggers, Thermal contact conductance characterization via computational contact homogenization: a finite deformation theory framework, International journal for numerical methods in engineering 83 (1) (2010) $27-58$.

[42] İ. Temizer, P. Wriggers, A multiscale contact homogenization technique for the modeling of third bodies in the contact interface, Computer Methods in Applied Mechanics and Engineering 198 (34) (2008) 377 - 396. doi:http://dx.doi.org/10.1016/j.cma.2008.08.008.

URL http://www.sciencedirect.com/science/article/pii/S0045782508003095

[43] D. E. Newland, An introduction to random vibrations, spectral \& wavelet analysis, Courier Corporation, 2012.

[44] M. Shinozuka, Simulation of multivariate and multidimensional random processes, The Journal of the Acoustical Society of America 49 (1B) (1971) 357-368. doi:http://dx.doi.org/10.1121/1.1912338. URL http://scitation.aip.org/content/asa/journal/jasa/49/1B/10.1121/1.1912338

[45] F. Poirion, C. Soize, Numerical methods and mathematical aspects for simulation of homogeneous and non homogeneous gaussian vector fields, in: P. Krée, W. Wedig (Eds.), Probabilistic Methods in Applied Physics, Vol. 451 of Lecture Notes in Physics, Springer Berlin Heidelberg, 1995, pp. 17-53. doi:10.1007/3-540-60214-3-50. URL http://dx.doi.org/10.1007/3-540-60214-3-50

[46] S. Cai, B. Bhushan, Meniscus and viscous forces during separation of hydrophilic and hydrophobic surfaces with liquidmediated contacts, Materials Science and Engineering: R: Reports 61 (1) (2008) 78-106.

[47] D. B. Asay, S. H. Kim, Evolution of the adsorbed water layer structure on silicon oxide at room temperature, The Journal of Physical Chemistry B 109 (35) (2005) 16760-16763. arXiv:http://pubs.acs.org/doi/pdf/10.1021/jp053042o, doi:10.1021/jp053042o.

URL http://pubs.acs.org/doi/abs/10.1021/jp053042o

[48] D. Maugis, B. Gauthier-Manuel, Jkr-dmt transition in the presence of a liquid meniscus, Journal of Adhesion Science and Technology 8 (11) (1994) 1311-1322. arXiv:http://dx.doi.org/10.1163/156856194X00627, doi:10.1163/156856194X00627. URL http://dx.doi.org/10.1163/156856194X00627

[49] B. Cappella, G. Dietler, Force-distance curves by atomic force microscopy, Surface science reports 34 (1) (1999) 1-104.

[50] R. Ardito, A. Frangi, F. Rizzini, A. Corigliano, Evaluation of adhesion in microsystems using equivalent rough surfaces modeled with spherical caps, European Journal of Mechanics - A/Solids (2015) doi:http://dx.doi.org/10.1016/j.euromechsol.2015.12.004. URL http://www.sciencedirect.com/science/article/pii/S0997753815001667

[51] V. A. Yastrebov, J. Durand, H. Proudhon, G. Cailletaud, Rough surface contact analysis by means of the finite element method and of a new reduced model, Comptes Rendus Mécanique 339 (78) (2011) 473 - 490, surface mechanics : facts and numerical models. doi:http://dx.doi.org/10.1016/j.crme.2011.05.006.

URL http://www.sciencedirect.com/science/article/pii/S163107211100091X

[52] F. W. DelRio, M. L. D. Dunn, M. P. de Boer, Capillary adhesion model for contacting micromachined surfaces, Scripta Materialia 59 (9) (2008) 916 - 920, viewpoint set no. 44 The materials for MEMS. doi:http://dx.doi.org/10.1016/j.scriptamat.2008.02.037.

URL http://www.sciencedirect.com/science/article/pii/S1359646208001668

[53] L. Kogut, I. Etsion, Elastic-plastic contact analysis of a sphere and a rigid flat, Journal of applied Mechanics 69 (5) (2002) $657-662$.

[54] L. Kogut, I. Etsion, Adhesion in elastic-plastic spherical microcontact, Journal of Colloid and Interface Science 261 (2) (2003) 372-378

[55] J. E. Jackson, A user's guide to principal components, Vol. 587, John Wiley \& Sons, 2005.

[56] I. Jolliffe, Principal component analysis, Wiley Online Library, 2002.

[57] I. K. Fodor, A survey of dimension reduction techniques (2002).

[58] S. Das, R. Ghanem, S. Finette, Polynomial chaos representation of spatio-temporal random fields from experimental mea- 
surements, Journal of Computational Physics 228 (23) (2009) 8726 - 8751. doi:http://dx.doi.org/10.1016/j.jcp.2009.08.025. URL http://www.sciencedirect.com/science/article/pii/S0021999109004677

[59] M. De Boer, T. Michalske, Accurate method for determining adhesion of cantilever beams, Journal of applied physics 86 (2) (1999) 817-827.

[60] A. Majumdar, C. Tien, Fractal characterization and simulation of rough surfaces, Wear 136 (2) (1990) 313-327.

[61] X. Xue, A. A. Polycarpou, L. M. Phinney, Measurement and modeling of adhesion energy between two rough microelectromechanical system (mems) surfaces, Journal of Adhesion Science and Technology 22 (5-6) (2008) 429-455.

[62] F. Yamazaki, M. Shinozuka, Digital generation of non-gaussian stochastic fields, Journal of Engineering Mechanics 114 (7) (1988) 1183-1197.

[63] F. W. DelRio, M. L. Dunn, B. L. Boyce, A. D. Corwin, M. P. De Boer, The effect of nanoparticles on rough surface adhesion, Journal of Applied Physics 99 (10) (2006) 104304.

[64] F. W. DelRio, M. L. Dunn, M. P. de Boer, Growth of silicon carbide nanoparticles using tetraethylorthosilicate for microelectromechanical systems, Electrochemical and solid-state letters 10 (1) (2007) H27-H30.

[65] K.-S. Kim, R. McMeeking, K. Johnson, Adhesion, slip, cohesive zones and energy fluxes for elastic spheres in contact, Journal of the Mechanics and Physics of Solids 46 (2) (1998) 243 - 266. doi:http://dx.doi.org/10.1016/S0022-5096(97)00070-7. URL http://www.sciencedirect.com/science/article/pii/S0022509697000707

[66] D. W. Scott, Multivariate density estimation: theory, practice, and visualization, John Wiley \& Sons, 2015.

67] D. Xiu, G. E. Karniadakis, The wiener-askey polynomial chaos for stochastic differential equations, SIAM journal on scientific computing 24 (2) (2002) 619-644.

[68] D. Xiu, Numerical methods for stochastic computations: a spectral method approach, Princeton University Press, 2010. 